\title{
Serotonin transporter variant drives preventable gastrointestinal abnormalities in development and function
}

\author{
Kara Gross Margolis, ${ }^{1}$ Zhishan Li, ${ }^{2}$ Korey Stevanovic, ${ }^{1}$ Virginia Saurman, ${ }^{1}$ Narek Israelyan, ${ }^{1,3}$ George M. Anderson, ${ }^{4}$ Isaac Snyder, \\ Jeremy Veenstra-VanderWeele, ${ }^{5}$ Randy D. Blakely, ${ }^{6}$ and Michael D. Gershon ${ }^{2}$ \\ 'Department of Pediatrics, ${ }^{2}$ Department of Pathology and Cell Biology, and I3nstitute of Human Nutrition, Columbia University College of Physicians and Surgeons, New York, New York, USA. \\ ${ }^{4}$ Child Study Center, Yale School of Medicine, New Haven, Connecticut, USA. ${ }^{5}$ Department of Psychiatry, Columbia University College of Physicians and Surgeons/New York Psychiatric Institute, \\ New York, New York, USA. ${ }^{6}$ Departments of Pharmacology and Psychiatry, Vanderbilt University, Nashville, Tennessee, USA.
}

\begin{abstract}
Autism spectrum disorder (ASD) is an increasingly common behavioral condition that frequently presents with gastrointestinal (GI) disturbances. It is not clear, however, how gut dysfunction relates to core ASD features. Multiple, rare hyperfunctional coding variants of the serotonin (5-HT) transporter (SERT, encoded by SLC6A4) have been identified in ASD. Expression of the most common SERT variant (Ala56) in mice increases 5-HT clearance and causes ASD-like behaviors. Here, we demonstrated that Ala56-expressing mice display GI defects that resemble those seen in mice lacking neuronal 5-HT. These defects included enteric nervous system hypoplasia, slow GI transit, diminished peristaltic reflex activity, and proliferation of crypt epithelial cells. An opposite phenotype was seen in SERT-deficient mice and in progeny of WT dams given the SERT antagonist fluoxetine. The reciprocal phenotypes that resulted from increased or decreased SERT activity support the idea that 5-HT signaling regulates enteric neuronal development and can, when disturbed, cause long-lasting abnormalities of $\mathrm{Gl}$ function. Administration of a 5- $\mathrm{HT}_{4}$ agonist to Ala56 mice during development prevented Ala56associated $\mathrm{GI}$ perturbations, suggesting that excessive SERT activity leads to inadequate $5-\mathrm{HT}_{4}$-mediated neurogenesis. We propose that deficient 5-HT signaling during development may contribute to $\mathrm{Gl}$ and behavioral features of ASD. The consequences of therapies targeting SERT during pregnancy warrant further evaluation.
\end{abstract}

\section{Introduction}

The increasingly frequent diagnosis of autism spectrum disorder (ASD) is based on deficits in social communication and interaction together with restricted, repetitive patterns of behavior (1). Although the core features of ASD are behavioral, gastrointestinal (GI) disturbances, particularly constipation, are 4-fold more common in ASD (2). Platelet serotonin (5-HT), moreover, which is primarily GI-derived (3), is increased in about one-third of patients with ASD $(4,5)$. Platelets do not synthesize 5-HT (6) but utilize the 5-HT transporter (SERT, encoded by Slc6a4) (7) to take it up (8) as they circulate through the gut. Multiple hyperactive coding variants of SERT have been found in subjects with ASD (9-11). Knockin mice expressing the most common of these, SERT Ala56 (12), display hyperserotonemia, increased 5-HT clearance, and hypersensitivity of central 5- $\mathrm{HT}_{1 \mathrm{~A}}$ and $5-\mathrm{HT}_{2 \mathrm{~A}}$ receptors, as well as repetitive behaviors and deficits of socialization that are reminiscent of ASD (13). The phenotype of SERT Ala56 mice suggests that the constitutively elevated activity of SERT in these animals interferes with the 5-HT signaling required for normal brain development and later function (14-16).

Neither 5-HT nor SERT is exclusive to the CNS. The bulk of 5 -HT in the body is found in the intestine, where it is a multifunc-

Conflict of interest: The authors have declared that no conflict of interest exists. Submitted: September 28, 2015; Accepted: March 3, 2016.

Reference information: J Clin Invest. 2016;126(6):2221-2235. doi:10.1172/JCI84877. tional signaling molecule during fetal and adult life (17). Changes in SERT-mediated 5-HT clearance, therefore, could contribute to GI and other peripheral comorbidities that accompany ASD. Enteric actions of 5-HT, like those in the brain, are terminated by SERT (18-20). In the bowel, moreover, enterocytes, as well as serotonergic neurons, express SERT $(18,19,21)$. SERT uptake into enterocytes and subsequent intracellular catabolism are also important in restricting the spread of mucosal 5-HT to, or desensitization of, aberrant targets.

Two 5-HT depots exist in the gut, each with its own biosynthetic enzyme $(22,23)$. A large tryptophan hydroxylase 1-dependent (TPH1-dependent) 5-HT pool exists in mucosal enterochromaffin (EC) cells and, in rats and mice, mast cells, whereas a smaller TPH2-dependent pool exists in the serotonergic neurons of the enteric nervous system (ENS). Both pools regulate GI motility $(22,24)$. EC cell 5-HT stimulates extrinsic sensory nerves $(25,26)$, which transmit signals of noxious stimuli $(27)$, discomfort, and pain to the CNS. 5-HT also stimulates intrinsic primary afferent neurons, which recruit the ENS to mediate peristaltic (24, 28-30) and secretory reflexes (31). Enteric neuronal 5-HT functions not only as a neurotransmitter but also as a growth factor, stimulating $5-\mathrm{HT}_{4}$ receptors that promote neurogenesis during development and adult life $(22,32)$. Neuronal 5-HT also regulates crypt epithelial cell proliferation (33).

The current study was undertaken to test the hypothesis that SERT Ala56 hyperactivity drives structural and functional GI def- 
icits due to altered 5-HT signaling that can be prevented through mechanism-guided interventions. We assessed the structure and function of the gut in SERT Ala56 animals, comparing results with those obtained in mice either lacking SERT or treated from gestation to weaning with the selective 5 -HT reuptake inhibitor fluoxetine. We detected significant, reciprocal abnormalities in these models related to enteric neuronal development, regulation of GI motility, and mucosal growth. Transcription of both Tph isoforms was also abnormal. The SERT Ala56-associated deficits were prevented if the animals were treated during development with a $5-\mathrm{HT}_{4}$ agonist, prucalopride (34). Our findings reveal that adult bowel function is exquisitely sensitive to SERT activity during ontogeny, and they confirm that 5-HT signaling is critical for the formation of the ENS. Therapies that perturb SERT activity during development, therefore, may have long-term consequences for bowel function. Observations also suggest that defective 5-HT signaling could provide a common mechanism contributing to peripheral and central defects in ASD.

\section{Results}

Numbers of enteric neurons are inversely related to SERT activity during development. 5-HT promotes enteric neuronal development (22, 32); therefore, we tested the hypothesis that SERT hyperactivity in SERT Ala56 mice would impede enteric neurogenesis (Figure 1). Parallel comparative experiments were carried out with Slc6a4 ${ }^{-/-}$ (herein referred to as SERTKO) animals (Figure 2) and mice exposed to the SERT antagonist fluoxetine from E1 to P21 (Figure 3). Total numbers of enteric neurons and subsets were identified immunocytochemically and quantified in whole mounts of laminar preparations dissected from the gut wall of mice at 6-8 weeks of age. Neuronal numbers were quantified as a function of ganglionic area, which is a parameter that is relatively resistant to stretching of the tissue (35). SERT Ala56 and SERTKO mice were compared with WT littermates. Fluoxetine-treated mice were compared with untreated control animals. Because serotonergic neurons are born early and 5-HT enhances development of late-born neurons expressing tyrosine hydroxylase $(\mathrm{TH})$ (dopaminergic neurons), $\gamma$-aminobutyric acid (GABA), and calcitonin gene-related peptide (CGRP) $(22,36,37)$, these phenotypes were chosen for study. GABA-expressing neurons were quantified in the myenteric plexus where they are located, whereas TH- and CGRP-expressing neurons were quantified in the submucosal plexus where most are found. Total enteric neurons were significantly less abundant in SERT Ala56 than in WT mice (Figure 1). This difference was observed in both the submucosal (Figure 1A; also compare I with J) and the myenteric plexus of the ileum (Figure 1E; also compare $\mathrm{O}$ with P) and colon (Figure 1F; also compare Q with R). In contrast, in SERTKO mice, total neurons were significantly more abundant in both the submucosal (Figure 2A; also compare $\mathrm{H}$ with I) and the myenteric plexus (Figure 2E; also compare J with $\mathrm{K}$ ) of the small intestines than in WT animals. The bowel of fluoxetine-treated mice was similar to that of SERTKO animals; again, more neurons were found in both the submucosal (Figure 3A; also compare $\mathrm{O}$ with R) and the myenteric plexus (Figure 3E; also compare I with L) of the fluoxetine-treated than the control mice.

Late-born neuronal phenotypes were more sensitive to the level of SERT activity during development than total neurons and thus appeared to be selectively affected. In SERT Ala56 mice, submucosal TH-expressing (Figure 1B; also compare K with L) and CGRP-expressing neurons (Figure 1C; also compare M with N) were each deficient, not only in absolute terms but as a proportion of total neurons (Figure 1D). In contrast, TH-expressing (Figure 2B; also compare P with Q) and CGRP-expressing neurons (Figure 2C; also compare $\mathrm{L}$ with $\mathrm{M}$ ) were overly abundant in the submucosal plexus of SERTKO animals, again as a proportion of total neurons (Figure 2D). Submucosal TH-expressing (Figure 3B; also compare $\mathrm{P}$ with S) and CGRP-expressing neurons (Figure 3C; also compare Q with T) were similarly hyperplastic in fluoxetine-treated mice, once more not only absolutely but also as a proportion of total neurons (Figure 3D). Similarly, SERT Ala56 mice were deficient in GABA-expressing neurons in the myenteric plexus of the ileum (Figure 1G; also compare $\mathrm{S}$ with $\mathrm{T}$ ) and colon (Figure $1 \mathrm{H}$; also compare $\mathrm{U}$ with $\mathrm{V}$ ), whereas GABA-expressing neurons were overly abundant in both SERTKO (Figure 2, F and G; also compare N and $\mathrm{R}$ with $\mathrm{O}$ and $\mathrm{S}$ ) and fluoxetine-treated mice (Figure 3, $\mathrm{G}$ and $\mathrm{H}$; also compare K with N). Expression of SERT Ala56 thus leads to a hypoplastic ENS with selective impairment of development/ survival of late-born neurons, the development of which is also 5-HT-promoted (22). In contrast, in SERTKO mice and in animals subjected to developmental fluoxetine exposure, the ENS is hyperplastic and the development/survival of those neurons that are impaired in SERT Ala56 mice is selectively enhanced.

The placenta has recently been found to be a transient source of 5-HT in early embryos that is able to influence CNS development prior to the endogenous synthesis of $5-\mathrm{HT}$ in central serotonergic neurons (38). The presence of 5-HT in early embryos thus makes it possible for the hyperfunctional SERT Ala56 mutation to decrease enteric neurogenesis even before $5-\mathrm{HT}$ is synthesized in the fetal gut. In fact, the density of serotonergic neurons in the SERT Ala56 intestine was only $36.3 \% \pm 5.6 \%(P<0.01 ; n=6)$ of that found in the intestines of WT littermates (see Supplemental Figure 4, A and B; supplemental material available online with this article; doi:10.1172/JCI84877DS1). In contrast, the density of serotonergic neurons in the intestines of fluoxetine-treated mice was significantly greater than that of untreated control animals (Figure 3, F, J, and M). The numbers of serotonergic neurons in SERTKO mice could not accurately be determined, because the content of the marker for these neurons, 5 -HT, is reduced (due to lack of reuptake) in neurons of SERTKO animals. Serotonergic neurites, however, were readily visible, and appeared to ramify more extensively in SERTKO than in either G56A or WT gut (Supplemental Figure 4, C and D).

Because of the ENS hyperplasia in SERTKO and fluoxetinetreated mice, transverse sections of the bowel were examined to determine whether neurons might be located ectopically in the mucosa or within smooth muscle coats, as occurs in mice lacking Hox11l1, which also display ENS hyperplasia (39). No ectopic neurons were found either in SERTKO or in fluoxetine-treated mice (not shown), suggesting that 5-HT affects neuronal development/survival but not the migration of neural precursors or patterns of gangliogenesis.

The SERT Ala56 mutation leads to slow GI transit and impairment of the peristaltic reflexes. Experiments were carried out to determine whether the long-lasting ENS hypoplasia associated 
A Submucosal plexus

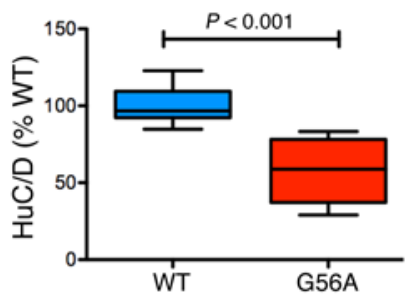

E

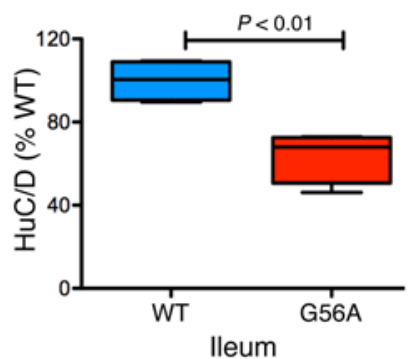

lleum
$\mathbf{B}$

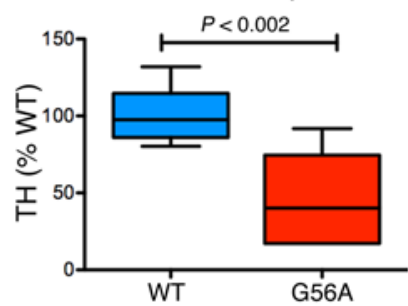

F

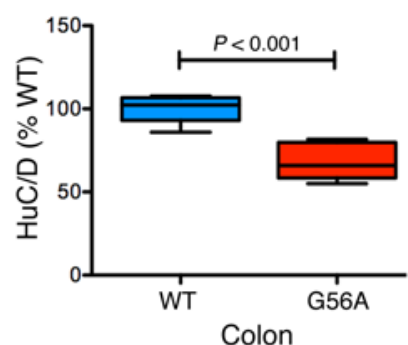

C

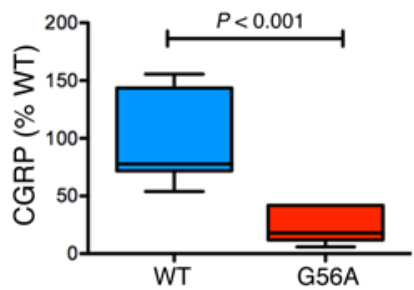

G

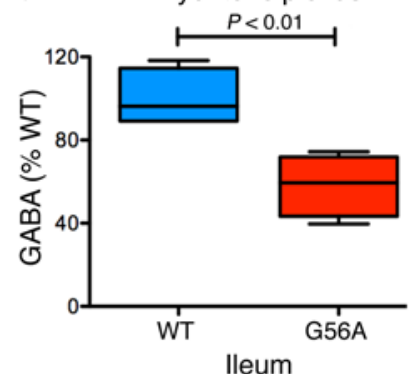

D Submucosal plexus

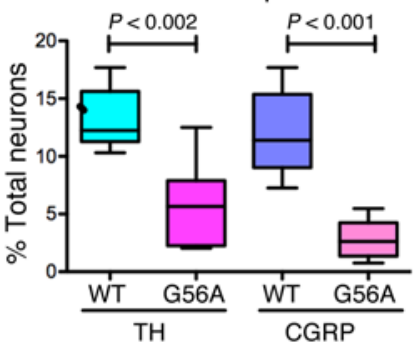

H Myenteric plexus

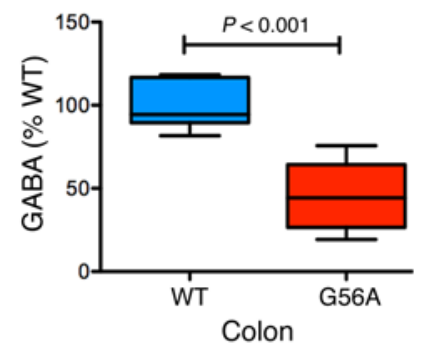

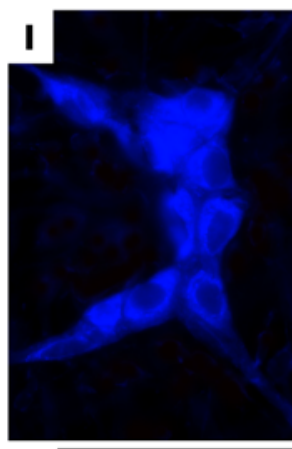

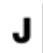

K

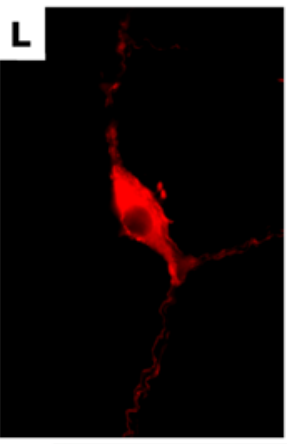

M

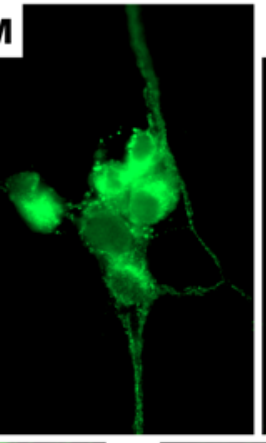

N
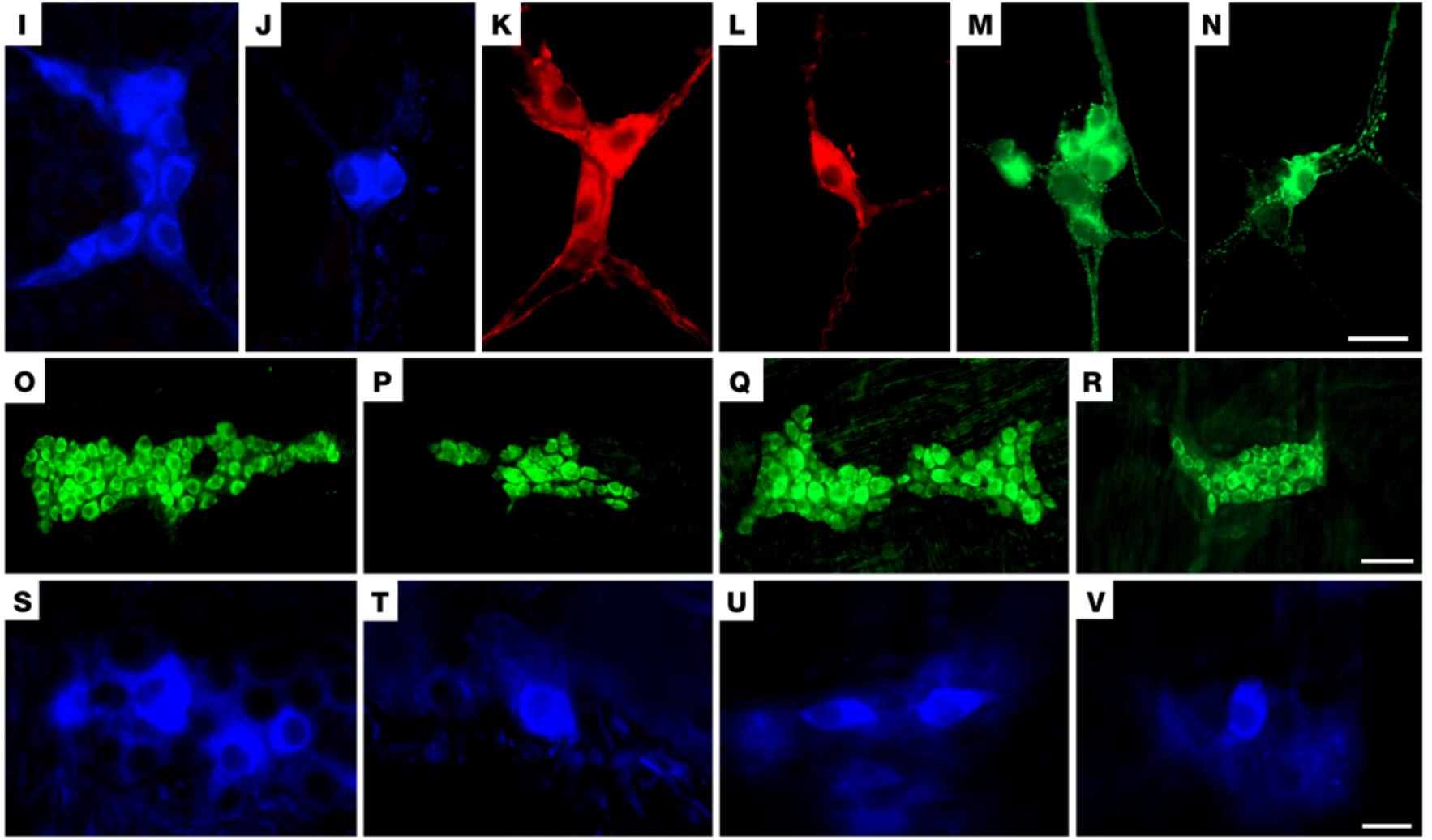

Figure 1. Numbers of total and late-born enteric neurons are lower in SERT Ala56 than in WT mice. $n=6-7 / g r o u p$. (A-D) Submucosal plexus, small intestine: (A) Total neurons. (B) Dopaminergic neurons (TH-immunoreactive). (C) CGRP-immunoreactive neurons. (D) Dopaminergic and CGRP-expressing neurons as a proportion of total neurons. (E-H) Myenteric plexus: (E) Total neurons, small intestine. (F) Total neurons, colon. (C) GABAergic neurons, small intestine. (H) GABAergic neurons, colon. (I and J) Submucosal HuC/D-immunoreactive neurons (blue): I shows WT; J shows SERT Ala56. (K and L) Submucosal TH-immunoreactive neurons (red): K shows WT; L shows SERT Ala56. (M and $\mathbf{N}$ ) Submucosal CGRP-immunoreactive neurons (green): $\mathbf{M}$ shows WT; $\mathbf{N}$ shows SERT Ala56. ( $\mathbf{O}$ and $\mathbf{P})$ Myenteric HuC/D-immunoreactive neurons, small intestine (green): $\mathbf{O}$ shows WT; $\mathbf{P}$ shows SERT Ala56. (Q and $\mathbf{R}$ ) Myenteric HuC/D-immunoreactive neurons, colon (green): $\mathbf{Q}$ shows WT; $\mathbf{R}$ shows SERT Ala56. (S and T) Myenteric GABA-immunoreactive neurons, small intestine (blue): $\mathbf{S}$ shows WT; T shows SERT Ala56. (U and V) Myenteric GABA-immunoreactive neurons, colon (blue): U shows WT; V shows SERT Ala56. Scale bars in $\mathbf{N}$ (applies to I-N), $\mathbf{R}$ (applies to $\mathbf{O}-\mathbf{R}$ ), and $\mathbf{V}$ (applies to $\mathbf{S}-\mathbf{V}$ ): $30 \mu \mathrm{m}$. Hu-C/D, pan-neuronal marker. 

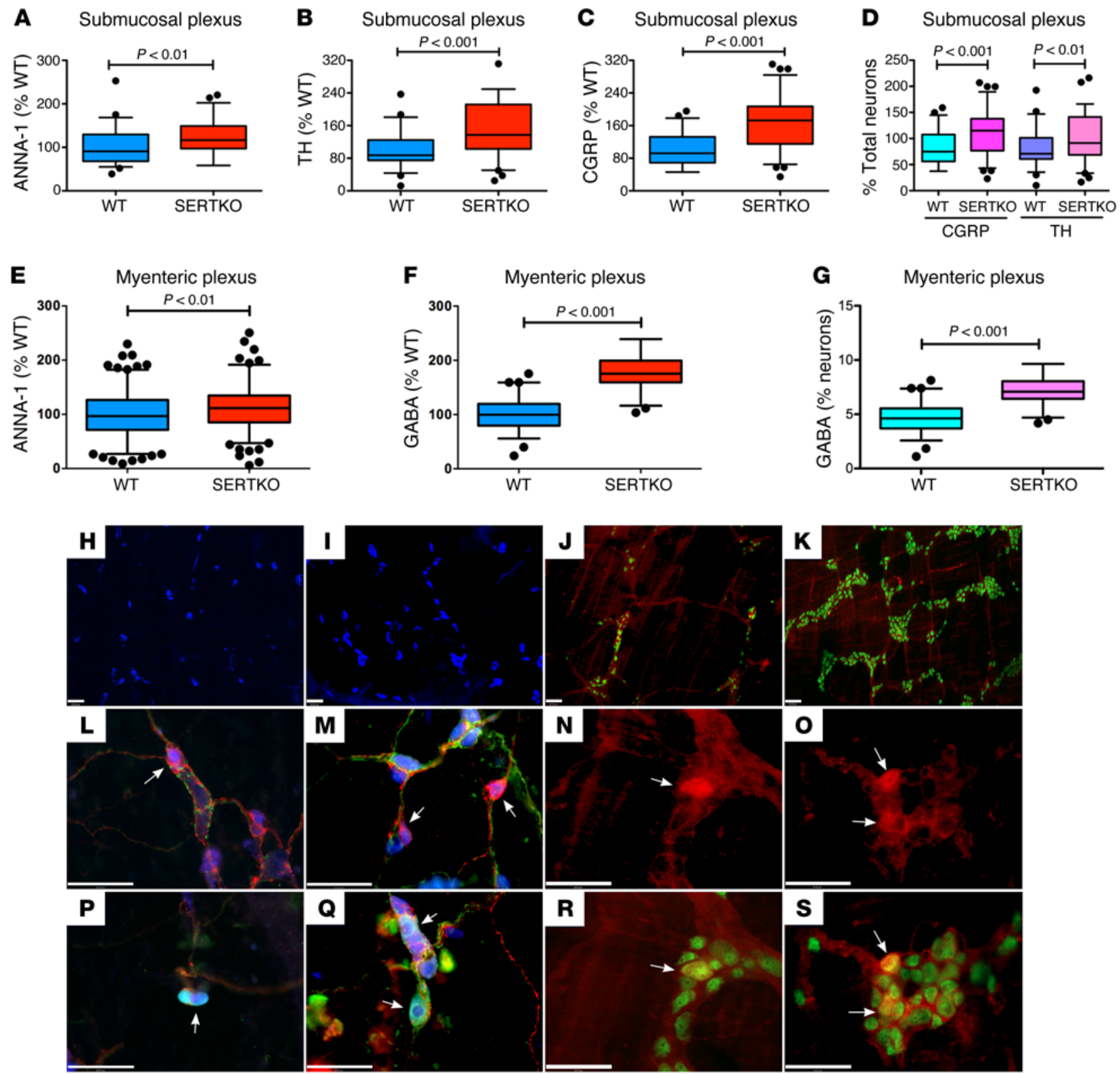

Figure 2. Numbers of total and late-born enteric neurons are greater in SERTKO than in WT mice. $n=6$. (A-D) Submucosal plexus, small intestine: (A) Total neurons. (B) Dopaminergic neurons (TH-immunoreactive). (C) CGRP-immunoreactive neurons. (D) Dopaminergic and CGRP-expressing neurons as a proportion of total neurons. (E-G) Myenteric plexus: (E) Total neurons, small intestine. (F) GABAergic neurons, small intestine. (G) GABAergic neurons as a proportion of total neurons. (H and I) Submucosal ANNA-1-immunoreactive neurons (blue): H shows WT; I shows SERTKO. (J and K) Myenteric ANNA-1immunoreactive neurons (green; red in background is due to GABA immunoreactivity): J shows WT; K shows SERTKO. (L and M) Submucosal CGRPimmunoreactive neurons (red [arrow]). The blue is due to ANNA-1 immunoreactivity in nerve cell bodies; note that CGRP-immunoreactive cells contain coincident ANNA-1 immunoreactivity. The green fibers are immunostained with Abs to TH. L shows WT; M shows SERTKO. (N and $\mathbf{0}$ ) Myenteric GABAimmunoreactive neurons (red [arrows]): $\mathbf{N}$ shows WT; $\mathbf{O}$ shows SERTKO. ( $\mathbf{P}$ and $\mathbf{Q}$ ) Submucosal TH-immunoreactive neurons (green [arrows]). The blue is due to ANNA-1 immunoreactivity in nerve cell bodies; note that TH-immunoreactive cells contain coincident ANNA-1 immunoreactivity. The red fibers that are also visible are immunostained with Abs to CGRP. P shows WT; $\mathbf{Q}$ shows SERTKO. ( $R$ and $\mathbf{S}$ ) Myenteric plexus doubly immunostained to reveal GABA (red [arrows]) and ANNA-1 (green) immunoreactivities. Note that all GABA-immunoreactive cell bodies, but none of the fiber tracts, display coincident ANNA-1 immunoreactivity. R shows WT; $\mathbf{S}$ shows SERTKO. These are the same fields illustrated in $\mathbf{N}$ and $\mathbf{0}$, but the merged image is shown to display CABA and ANNA-1 immunoreactivities simultaneously. Scale bars: $50 \mu \mathrm{m}$.

with the SERT Ala56 mutation is reflected in GI motility. Mice were examined at 6-8 weeks of age. Measurements were made in vivo of total GI transit time, gastric emptying, small intestinal transit (SIT), and propulsive colorectal motility. Colonic migrating motor complexes (CMMCs; peristaltic reflexes) were also investigated in vitro to determine whether changes in intestinal transit in SERT Ala56 mice are due to a defect that is intrinsic to the ENS. The extrinsic innervation of the gut is severed in isolated preparations, and the intrinsic circuits of the ENS mediate CMMCs (40). 

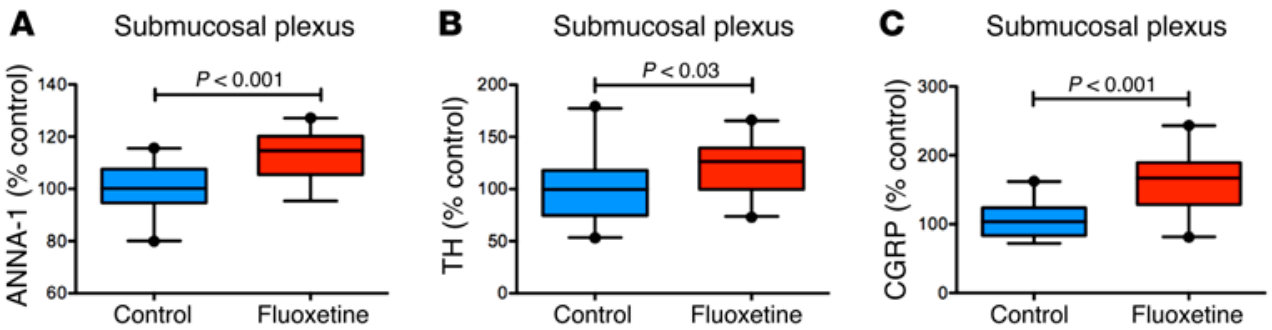

D Submucosal plexus

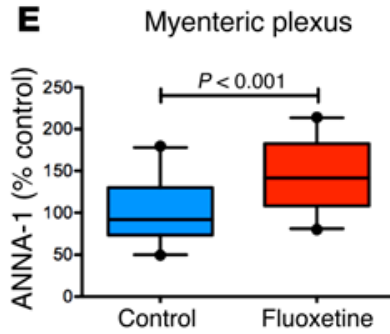

$\mathbf{F}$

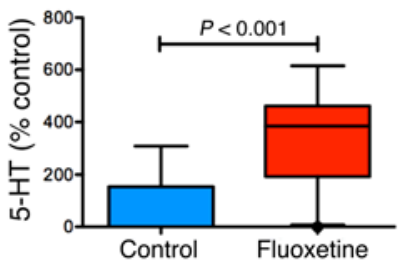

G

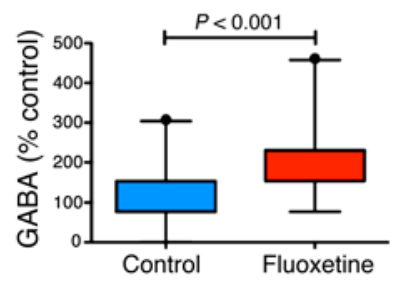

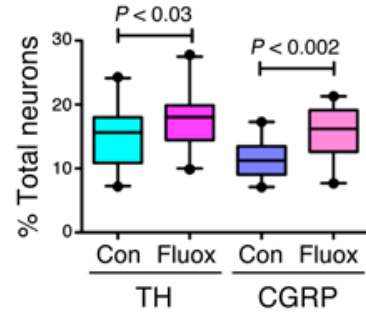

H Myenteric plexus

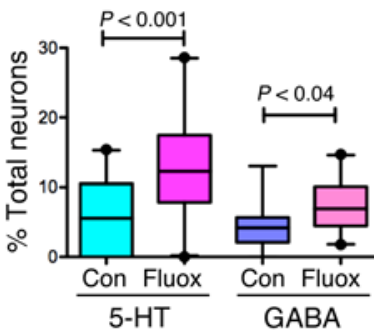

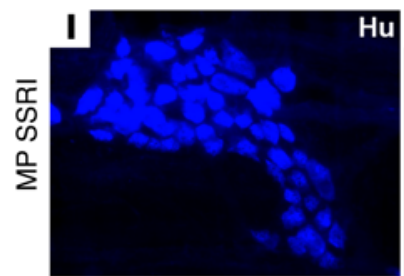
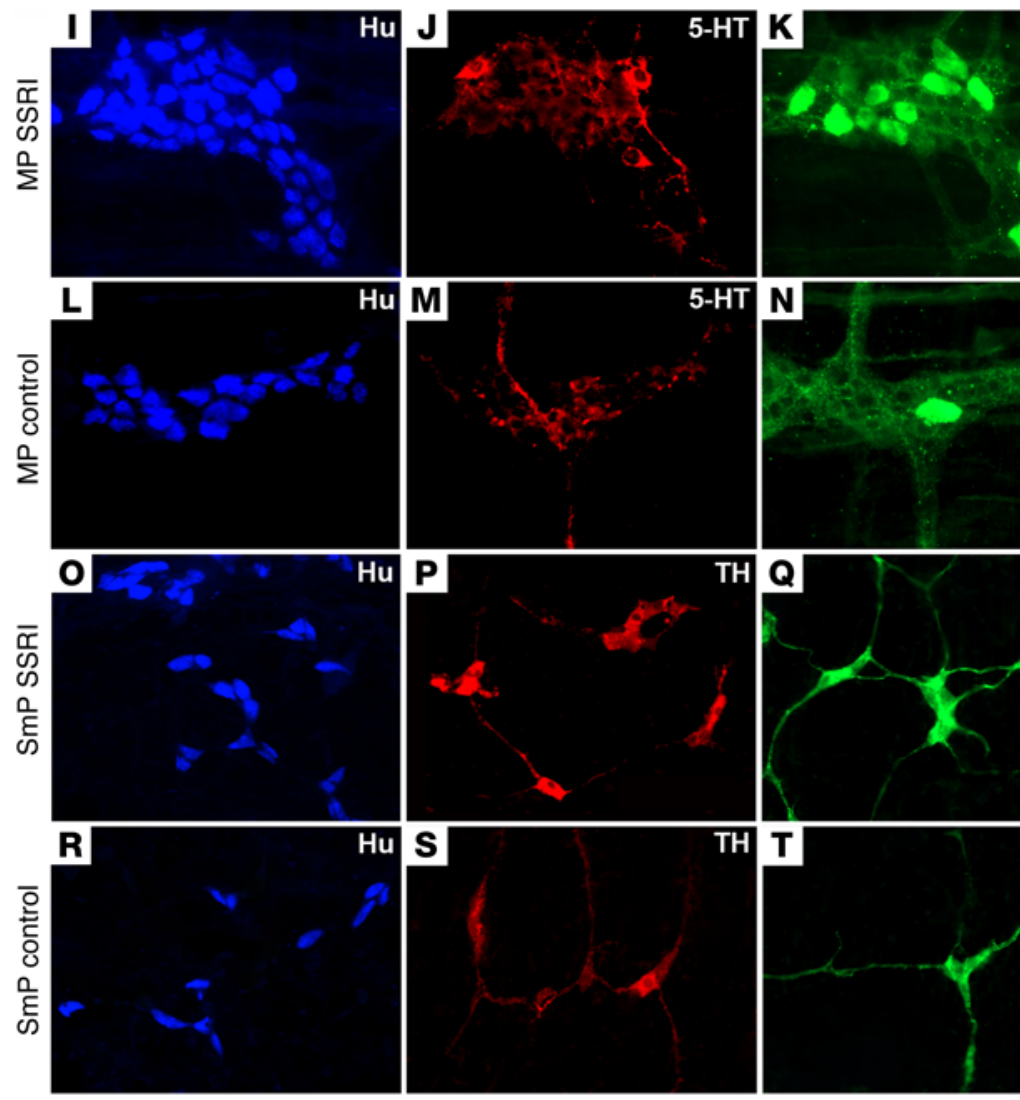
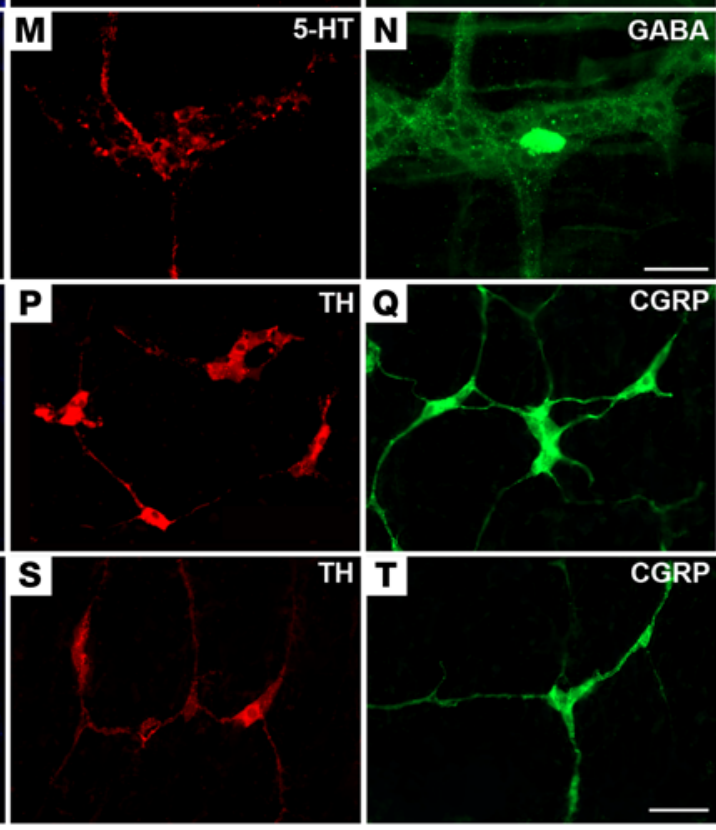

Figure 3. Numbers of total and late-born enteric neurons are greater in mice exposed to fluoxetine during development than in control mice exposed identically during development to vehicle. $n=6$. (A-D) Submucosal plexus, small intestine: (A) Total neurons. (B) TH-immunoreactive neurons. (C) CGRP-immunoreactive neurons. (D) TH- and CCRP-expressing neurons as a proportion of total neurons. (E-H) Myenteric plexus: (E) Total neurons, small intestine. (F) 5-HT-immunoreactive neurons. (C) GABAergic neurons. (H) Serotonergic and CABAergic neurons as a proportion of total neurons. (I-K) Myenteric plexus (MP) of fluoxetine-treated mice. (I) Total neurons (ANNA-1-immunoreactive; blue). (J) 5-HTimmunoreactive neurons (red). (K) GABAergic neurons (green). (L-N) Myenteric plexus, control mice. (L) Total neurons (ANNA-1-immunoreactive; blue). (M) 5-HT-immunoreactive neurons (red). (N) GABAergic neurons (green). (0-Q) Submucosal plexus (SmP), fluoxetine-treated mice. (0) Total neurons (ANNA-1-immunoreactive; blue). (P) TH-immunoreactive neurons (red). (Q) GABAergic neurons (green). (R-T) Submucosal plexus, control mice. (R) Total neurons (ANNA-1-immunoreactive; blue). (S) TH-immunoreactive neurons (red). (T) CABAergic neurons (green). Scale bar for all myenteric images (I-N): $50 \mu \mathrm{m}$. Scale bar for all submucosal images (O-T): $25 \mu \mathrm{m}$.
Total GI transit time in SERT Ala56 mice was significantly longer than that of WT animals (Figure $4 \mathrm{~A} ; P<0.01$ ); however, the rate of gastric emptying (Figure 4B) and SIT (not illustrated) in SERT Ala56 and WT animals did not differ significantly. In contrast to the stomach and SIT, propulsive colorectal motility was significantly slower in SERT Ala56 than in WT mice (Figure 2C; $P<0.01$ ). Exogenous 5-HT administration increases motility in the small intestine of humans and lower animals $(41,42)$. We therefore compared SIT after exogenous $5-\mathrm{HT}$ injection $(1.0 \mathrm{mg} / \mathrm{kg})$ in WT and
SERT Ala56 mice. SIT was significantly slower in SERT Ala56 than in WT mice after the injection of exogenous 5-HT (Figure 4D), consistent with the hypothesis that the SERT Ala56 is hyperactive and reduces enteric effects even of exogenous 5-HT in vivo.

Intraluminal pressure was raised in isolated preparations of colon to initiate CMMCs, preparations were video-imaged, and spatiotemporal maps of contractile activity patterns were constructed (Figure 4, E and F). Analysis of these maps revealed that CMMC frequency (Figure 4G; $P<0.0001$ ), velocity (Figure $4 \mathrm{H}$; 

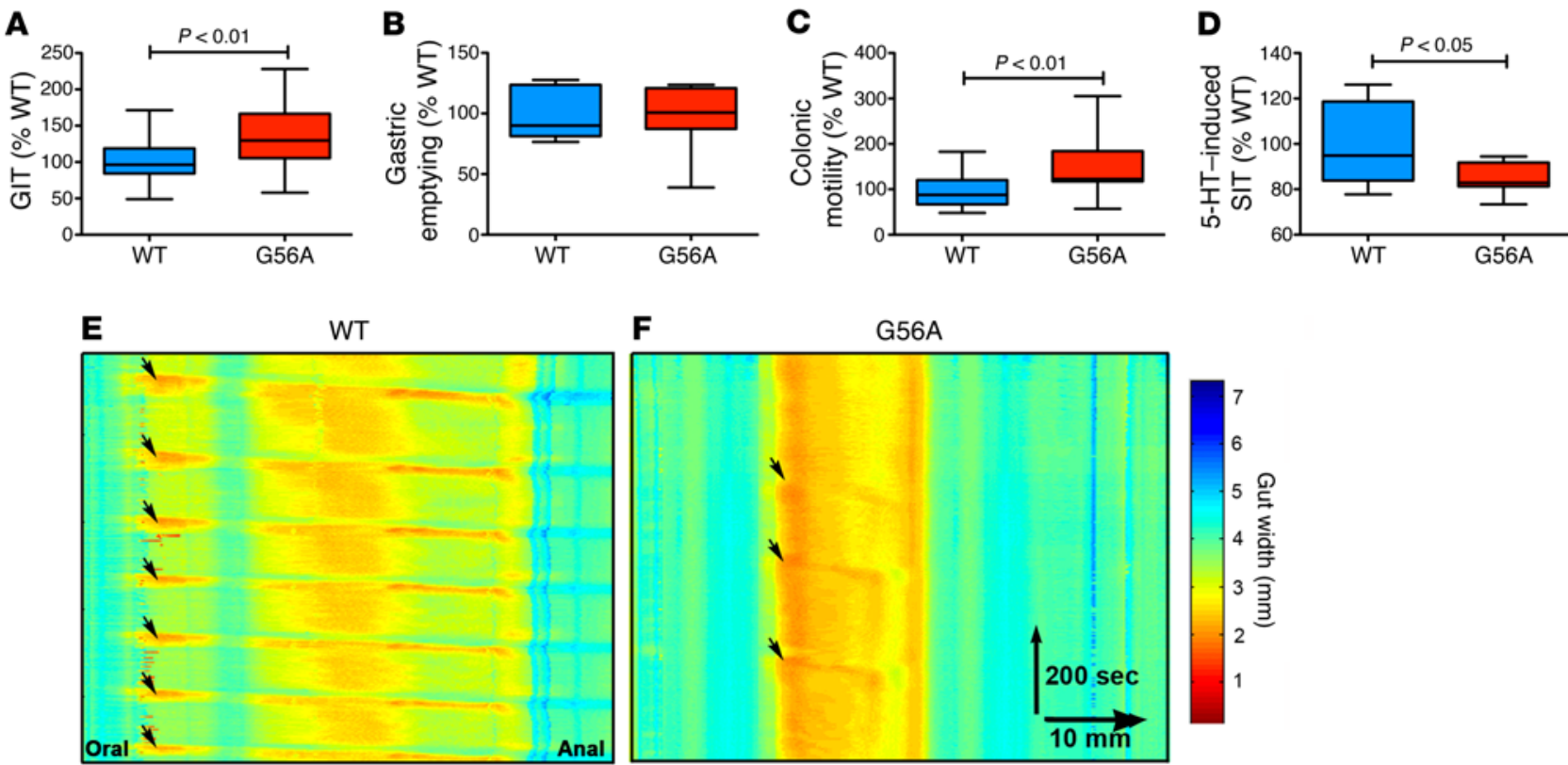

$\mathbf{F}$

G56A
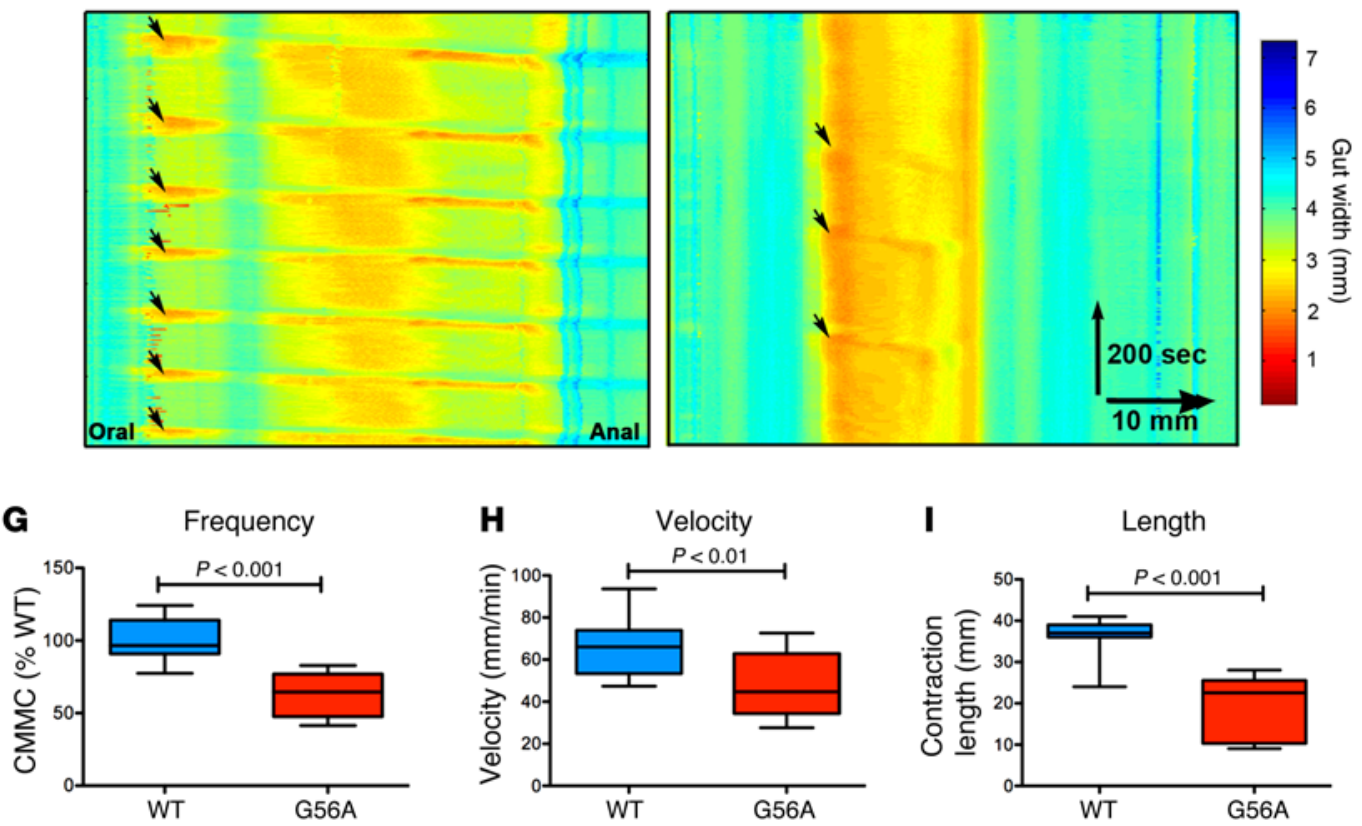

Figure 4. Intestinal motility is abnormally slow in SERT Ala56 mice. Intestinal motility was abnormally slow in SERT Ala56 mice ( $n=30-36 /$ group; 2 trials). (A) Total GI transit time (GIT) measured in vivo. (B) Gastric emptying. (C) Time to eject a bead from the rectum (colonic motility). (D) Small intestinal transit after a bolus injection of 5-HT. (E and F) WT (E) and SERT Ala56 (F) mice. Typical spatiotemporal maps showing CMMCs (arrows) in isolated preparations of colon ( $n=3-4$ per group). The ordinate represents time, and the abscissa represents oral-to-anal distance. The width of the gut (mm), indicative of contractions, was pseudocolored. (C) CMMC frequency. (H) CMMC velocity. (I) CMMC length of propagation. Student's unpaired $t$ test was used to compare groups. For the box-and-whisker plot, the boxes represent the first and third quartiles, the whiskers are $95 \%$ confidence interval, and the lines within the boxes are median values.

$P<0.01$ ), and length of propagation (Figure $4 \mathrm{I} ; P<0.001$ ) were all significantly lower in SERT Ala56 than in WT mice. These observations suggest that the ENS hypoplasia of SERT Ala56 mice impairs generation and conduction of peristaltic reflexes.

GI motility was analyzed in SERTKO mice and in animals given fluoxetine during development to compare the effects of the SERT Ala56-associated ENS hypoplasia with those of ENS hyperplasia. In vivo measurements of GI motility in SERTKO mice, however, are complicated by the desensitization of 5-HT receptors that has been found to occur spontaneously in these animals (18). Diarrhea and constipation thus alternate in individual SERTKO mice. In contrast to SERT Ala56 animals, total GI transit time (Figure 5A) in SERTKO mice was not significantly different from that of WT animals, although gastric emptying was also not significantly different (Figure 5B). Surprisingly, as in SERT Ala56 mice, SIT (Figure 5C) and propulsive colorectal motility (Figure 5D) were significantly slower in SERTKO than in WT mice. Parameters of in vivo GI transit in mice exposed to fluoxetine during development, moreover, slowed even more than they did in SERTKO animals. Total intestinal transit time in fluoxetine-treated animals was significantly increased (Figure 6A), SIT was significantly slower (Figure 6B), and the time required to eject beads from the rectum was significantly greater (Figure 6C) than in vehicle-treated control mice.

We tested the idea that the unexpectedly slow transit in SERTKO and fluoxetine-treated mice was due to enhanced sympathetic input from the CNS. This possibility is plausible because SERTKO mice are highly vulnerable to stress (43) and sympathetic stimulation, which slows GI transit, might result from the stress of measuring GI motility. We thus reinvestigated GI transit after chemical sympathectomy with 6-hydroxydopamine (6-OHDA) and also analyzed ENS-dependent CMMCs in isolated colon, which lacks a functional sympathetic innervation. Although sympathectomy accelerated transit, GI transit time and gastric emptying were not significantly different in WT and SERTKO mice (Figure 5, A and B). Sympathectomy, however, normalized SIT (Figure 5C) and propulsive colorectal motility (Figure 5D) in SERTKO mice. Similarly, sympathectomy eliminated the slowing of all measures of in vivo motility in fluoxetine-treated mice without affecting any of these parameters in matched control animals (Figure 6, A-C). These observations suggest that sympathetic slowing of GI 
A

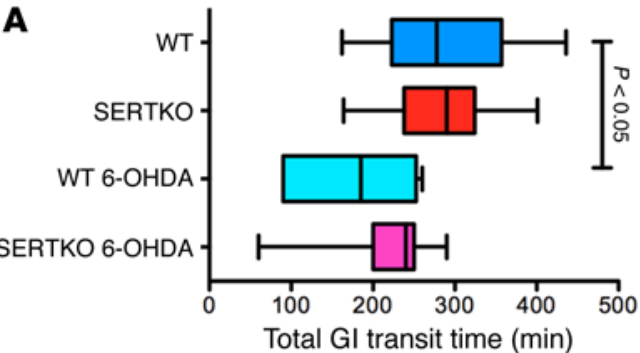

C

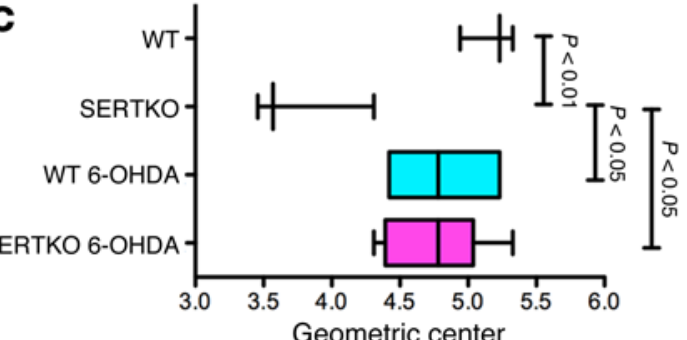

$\mathbf{E}$

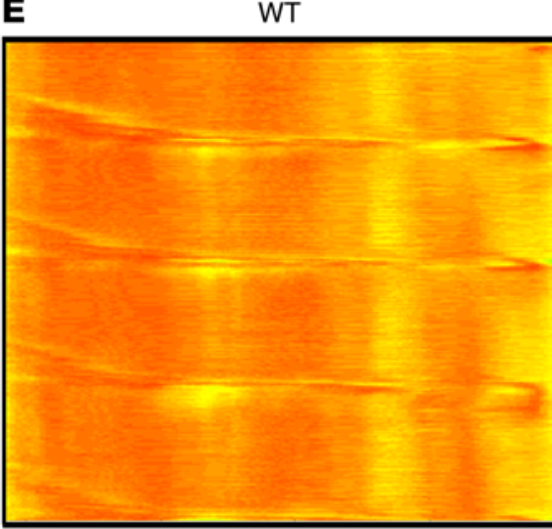

B

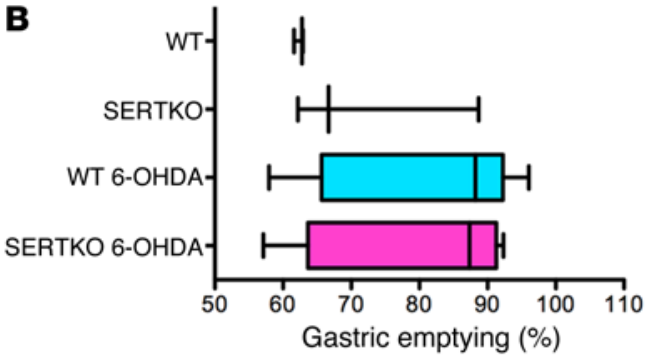

D

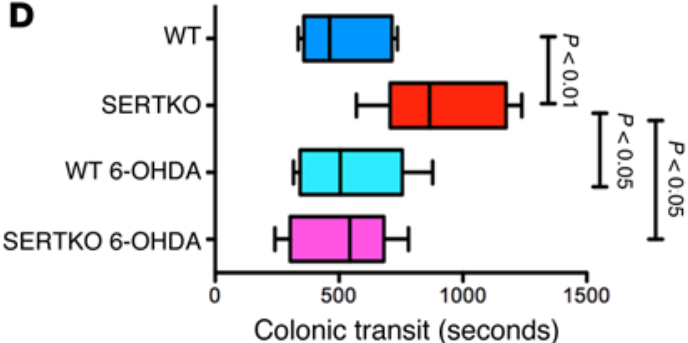

F SERTKO
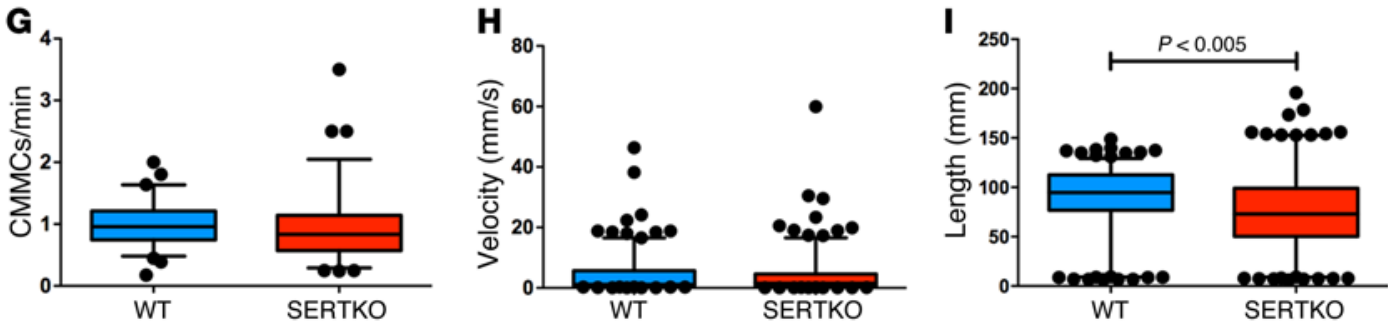

Figure 5. Sympathetic hyperactivity slows intestinal motility in SERTKO mice. Sympathetic hyperactivity slowed intestinal motility in SERTKO mice ( $n=6-9 /$ group for in vivo motility, 2 trials, and 12/group for peristalsis). (A) GIT measured in vivo. Total GI transit in WT and SERTKO mice was not significantly different. 6-OHDA accelerated transit in both. (B) Gastric emptying. SERTKO and WT mice were not significantly different. (C) Small intestinal transit. Transit was significantly slower in SERTKO than in WT mice. 6-OHDA did not affect transit in WT mice but eliminated slow transit in SERTKO mice. (D) Colonic motility. More time was required for bead ejection in SERTKO than in WT mice. 6-OHDA did not affect motility in WT mice but eliminated slow bead ejection in SERTKO animals. (E and F) WT (E) and SERT Ala56 (F). Typical spatiotemporal maps showing CMMCs in isolated colon as in Figure 4. (G) CMMC frequency. (H) CMMC velocity. (I) CMMC length of propagation. Student's unpaired t test and 1-way ANOVA were used, respectively, to compare single and multiple means. For the box-and-whisker plot, the boxes represent the first and third quartiles, the whiskers are $95 \%$ confidence interval, and the lines within the boxes are median values.

transit occurs and that both the deletion of SERT and its inhibition during development increase sympathetic input to the intestine. Analyses of the ENS-dependent CMMC in isolated preparations of colon from WT (Figure 5E), SERTKO (Figure 5F), control (Figure 6D), and fluoxetine-treated mice (Figure 6E) supported these ideas. CMMC frequency (Figure 5G) and velocity (Figure 5H) in
SERTKO were not slower than in WT mice, although the propagation length (Figure 5I) of CMMCs was significantly shorter. In isolated preparations of colon from mice that were exposed to fluoxetine during development, moreover, CMMC frequency (Figure 6F), velocity (Figure 6G), and propagation length (Figure $6 \mathrm{H})$ were all significantly greater than in those from control ani- 
A

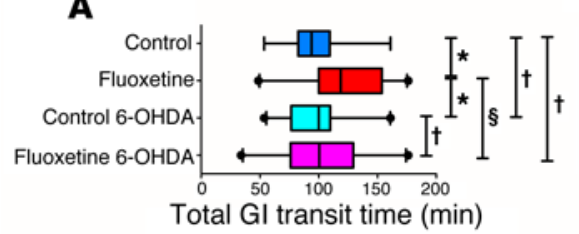

D

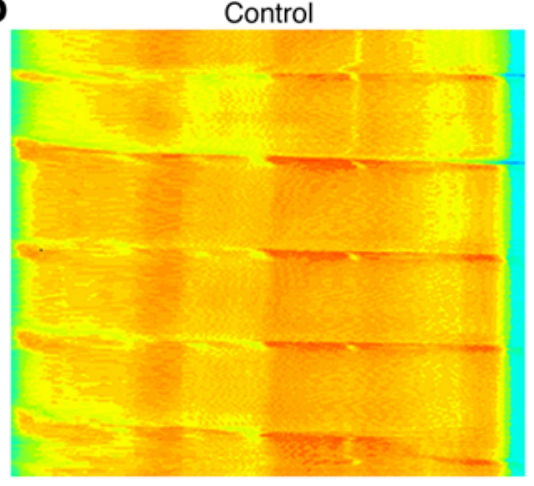

B

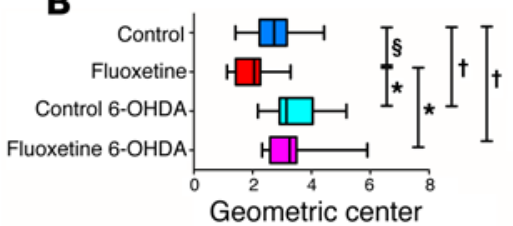

\section{C}

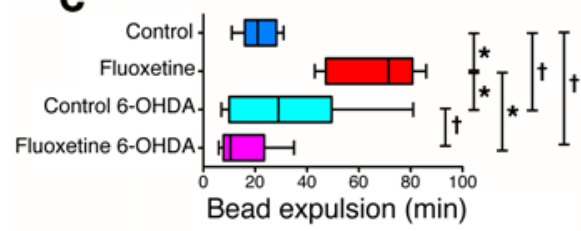

E

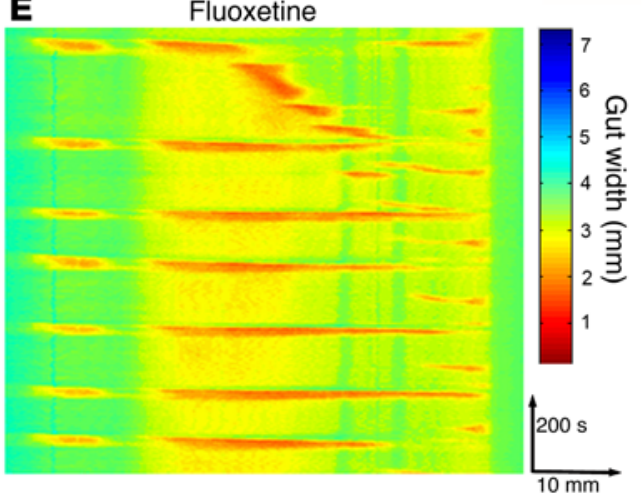

$\mathbf{F}$

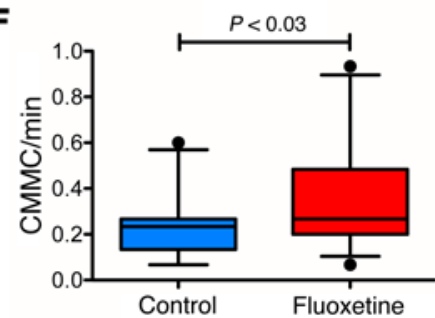

G

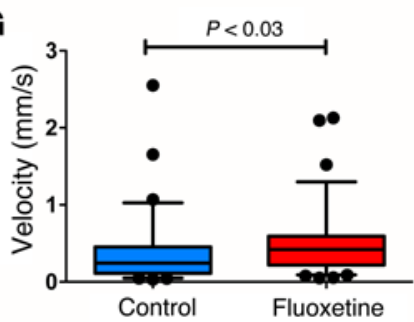

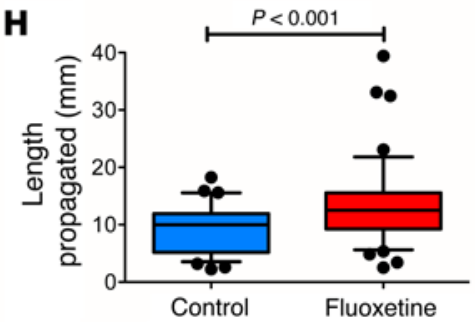

Figure 6. Chemical sympathectomy prevents in vivo slow intestinal transit of fluoxetine-treated mice. Chemical sympathectomy prevented in vivo slow intestinal transit in fluoxetine-treated mice ( $n=8-13$ /group for in vivo motility assays, 2 trials; $4 /$ group for peristalsis). Control and fluoxetine-treated animals were treated, respectively, with vehicle or 6-OHDA. Mice were examined within 2 weeks after the last injection. Data are from 4 mice of each type. (A) Total GIT. Fluoxetine treatment caused slow GIT. 6-OHDA corrected the defect in fluoxetine-treated mice but did not alter total GIT in control mice. (B) Small intestinal transit (SIT). Fluoxetine-treatment caused slow SIT. 6-OHDA corrected the deficit in fluoxetine-treated mice but did not affect SIT in control animals. (C) Colonic motility. Fluoxetine treatment increased the bead ejection time. 6-OHDA corrected the deficit in fluoxetine-treated mice but did not significantly alter ejection time in control animals. ${ }^{\circledR} P<0.05$; ${ }^{*} P<0.001 ;{ }^{\dagger} P$ was NS. (D and E) WT (D) and fluoxetine-treated (E) mice. Typical spatiotemporal maps showing CMMCs in isolated colon. (F) CMMC frequency. (C) CMMC velocity. (H) CMMC length of propagation. Student's unpaired $t$ test and 1-way ANOVA were used, respectively, to compare single and multiple means. For the box-and-whisker plot, the boxes represent the first and third quartiles, the whiskers are $95 \%$ confidence interval, and the lines within the boxes are median values.

mals. These observations suggest that ENS hyperplasia in mice exposed during development to fluoxetine is associated with facilitated generation, conduction, and propagation of peristaltic reflexes, which is the opposite of the effect of the ENS hypoplasia occurring in SERT Ala56 mice. Conceivably, the effects of SERT inhibition during development and SERT knockout are different because SERT is functional at the time that CMMCs are analyzed in fluoxetine-treated but not in SERTKO animals. SERT may be required during the generation of CMMCs to inactivate 5-HT, which participates in peristaltic reflex mediation (44). Spontaneous receptor desensitization in SERTKO mice (18) may also interfere with CMMCs. In contrast, chemical sympathectomy prevents slow in vivo transit in both SERTKO and fluoxetine-treated mice, suggesting that enhanced sympathetic input to the bowel occurs in each and thus is probably due to a developmental disturbance of sympathetic control in the CNS.

Administration of a 5-HT ${ }_{4}$ agonist during development rescues mice from the abnormalities of the ENS and GI motility associated with the SERT Ala56 mutation. 5- $\mathrm{HT}_{4}$ agonists increase enteric neurogenesis, and postnatal enteric neurogenesis is deficient in 5- $\mathrm{HT}_{4} \mathrm{KO}\left(\mathrm{Htr}^{--}\right)$mice (32). If SERT Ala56 hyperactivity were to impede enteric neurogenesis by diminishing $5-\mathrm{HT}$ availability at $5-\mathrm{HT}_{4}$ receptors, an exogenously supplied $5-\mathrm{HT}_{4}$ agonist that is not a substrate for SERT ought to countermand the defect. We tested this hypothesis by administering the $5-\mathrm{HT}_{4}$ agonist prucalopride $(15 \mathrm{mg} / \mathrm{kg})$, which was given orally to dams from gestation through weaning. Offspring were then allowed to mature without further treatment. Treatment with prucalopride was found to prevent the hypoplasia that occurs in total neurons (ANNA-1-immunoreactive) of SERT Ala56 mice in both the submucosal (Figure $7 \mathrm{~A}$ ) and the myenteric plexus (Figure 7D). Prucalopride treatment also prevented the SERT Ala56-associated deficiencies of the submucosal late-born submucosal dopaminergic (TH-immunoreactive; Figure 7B) and CGRP-immunoreactive neurons (Figure 7C) as well as the SERT Ala56-associated deficiency of the myenteric late-born GABA-immunoreactive neurons (Figure 7E). Prucalo- 
A Total submucosal neurons

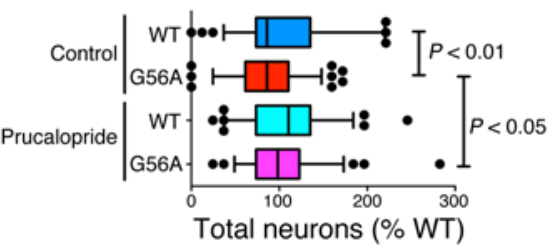

C
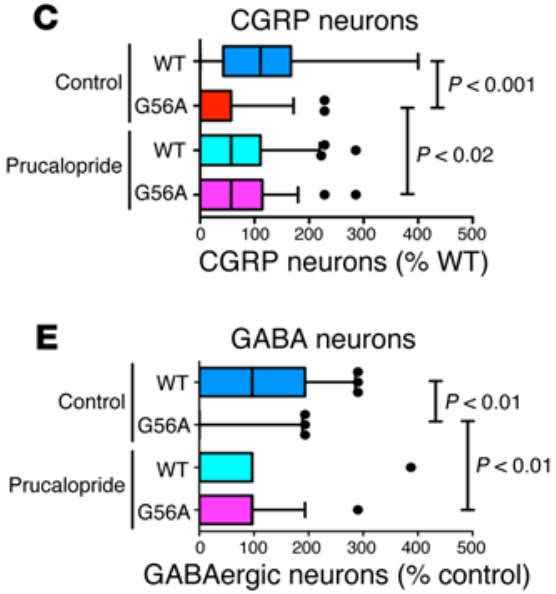

G Rectal bead ejection time

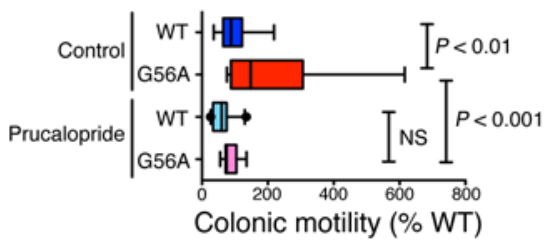

I

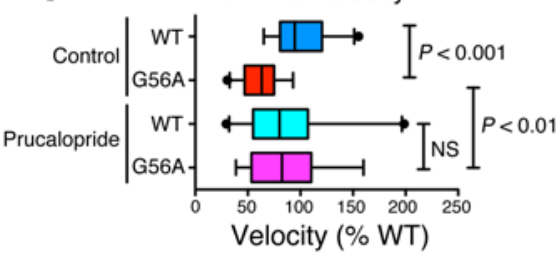

B

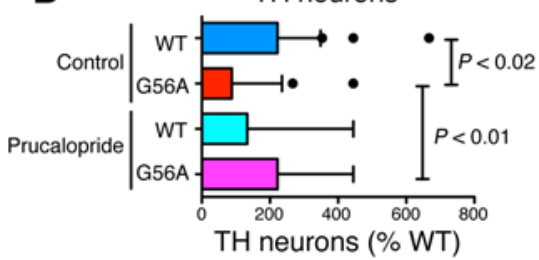

D Total myenteric neurons
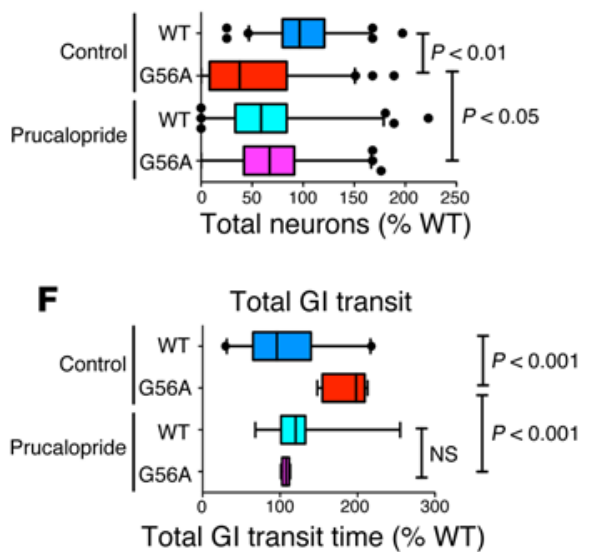

H

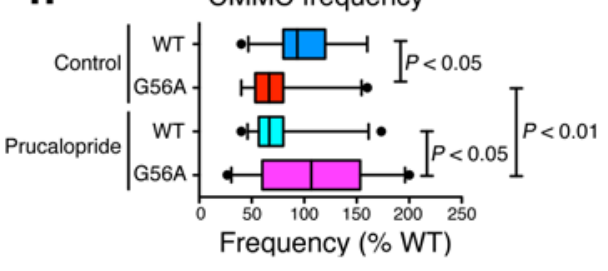

J

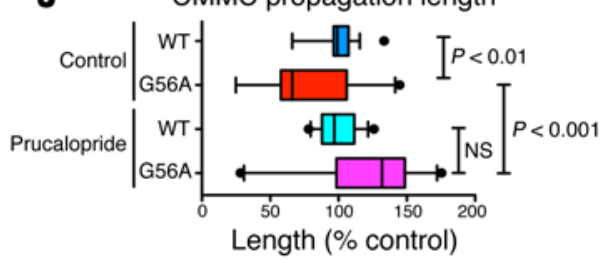

Figure 7. Prucalopride treatment during development rescues SERT Ala56 mice from ENS hypoplasia and associated abnormalities of GI motility. Treatment with prucalopride during development rescued SERT Ala56 mice from ENS hypoplasia ( $n=3-4 /$ group) and associated abnormalities of GI motility ( $n=14-36 /$ group for in vivo motility studies, 2 trials; 3-4/group for peristalsis). (A-C) Submucosal plexus. (A) Total neurons. Fewer neurons were found in SERT Ala56 than in WT mice. Prucalopride did not affect the number of neurons in WT animals but eliminated hypoplasia in SERT Ala56 mice. (B) TH-immunoreactive neurons. Prucalopride prevented the deficiency in SERT Ala56 mice. (C) CGRP-immunoreactive neurons. Prucalopride prevented the deficiency in SERT Ala56 mice. (D and E) Myenteric plexus. Total neurons (D) and GABAergic neurons (E) were fewer in SERT Ala56 mice than in WT mice. Numbers of total and GABAergic neurons were normalized by prucalopride administration to SERT Ala56 mice. (F) Total GIT was longer in SERT Ala56 mice than in WT mice. Prucalopride eliminated slow transit in SERT Ala56 mice but did not affect total GIT when given similarly to WT mice. (C) Colonic motility was significantly longer in SERT Ala56 than in WT mice. Prucaloparide eliminated this defect in SERT Ala56 animals but did not significantly affect colonic motility when given to WT mice. $(\mathbf{H}-\mathbf{J})$ CMMCs in isolated colon. Frequency $(\mathbf{H})$, velocity (I), and conduction length (J) were all lower in SERT Ala56 mice than in WT mice. Prucalopride eliminated each of these defects when given to SERT Ala56 mice but did not significantly alter CMMC parameters when given to WT animals. Student's unpaired $t$ test and 1-way ANOVA were used, respectively, to compare single and multiple means. For the box-and-whisker plot, the boxes represent the first and third quartiles, the whiskers are $95 \%$ confidence interval, and the lines within the boxes are median values. pride rescue of neuronal numbers was accompanied by elimination of the SERT Ala56-associated slowing of total GI transit time (Figure 7F) and of colonic motility (Figure 7G). The prucalopridemediated rescue of in vivo motility was also manifest in a rescue of CMMCs measured in vitro; prucalopride treatment prevented the SERT Ala56-associated decreases in CMMC frequency (Figure $7 \mathrm{H}$ ), velocity (Figure 7I), and length of propagation (Figure 7J). These in vitro observations suggest that prucalopride rescue is an intrinsic property of the ENS and is not mediated through altered CNS control of the gut. Interestingly, administration of prucalopride to WT mice during development led to a decrease in numbers of GABAergic neurons (Figure 7E) and a decrease in CMMC frequency (Figure $7 \mathrm{H}$ ) that were not seen when SERT Ala56 pups were exposed to prucalopride. These observations are consistent with the possibility that excessive stimulation of $5-\mathrm{HT}_{4}$ receptors, as might occur when prucalopride adds to endogenously released 5-HT, can lead to receptor desensitization or, alternatively, to overstimulation and neurotoxicity.
The SERT Ala56 mutation decreases crypt epithelial cell proliferation, stunts growth of villi, and decreases mucosal permeability. The ENS, and specifically 5-HT, have been linked to mucosal maintenance (33); myenteric serotonergic neurons innervate submucosal cholinergic neurons that regulate proliferation of transit-amplifying cells. As a result, the cell proliferation index, crypt depth, and villus height are all greater in SERTKO than in WT mice. Neuronal, rather than mucosal, 5-HT mediates these effects; SERTKO and double knockout SERTKO/TPH1KO (Slc6a4 $\left.4^{-/} \mathrm{Tph1}^{-/-}\right)$mice are equivalent, but enhanced mucosal growth does not occur in dou-

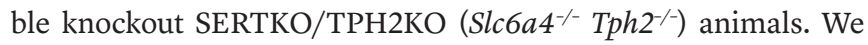
therefore compared villus height, crypt depth, and proliferation in SERT Ala56, SERTKO, and fluoxetine-treated mice (Figure 8 and Supplemental Figure 3).

Small intestinal villus height (Figure 8A) and crypt depth (Figure 8B) were both significantly less in SERT Ala56 mice than in WT littermates (Supplemental Figure 3, A and B). In contrast, in fluoxetine-treated mice, small intestinal villus height (Figure $8 \mathrm{C}$ ) 
A
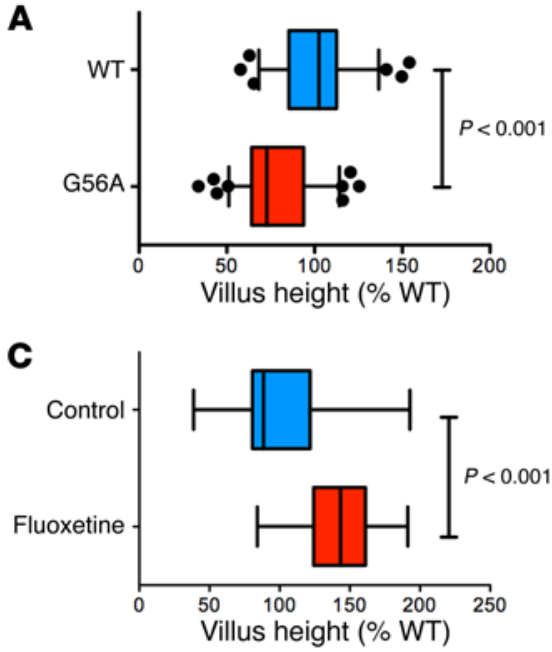

E

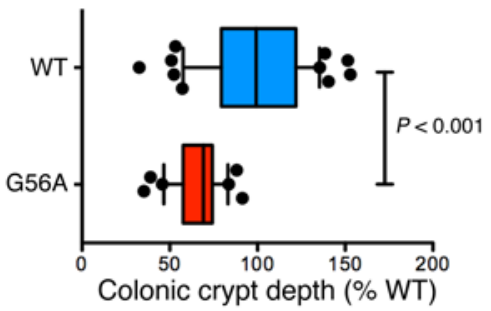

G

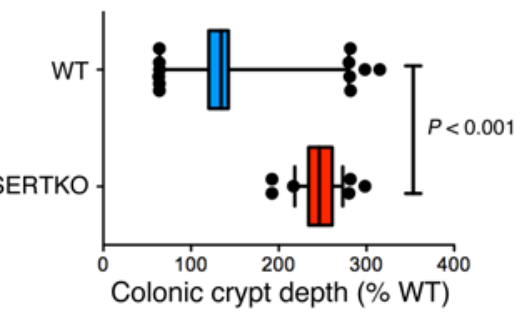

B

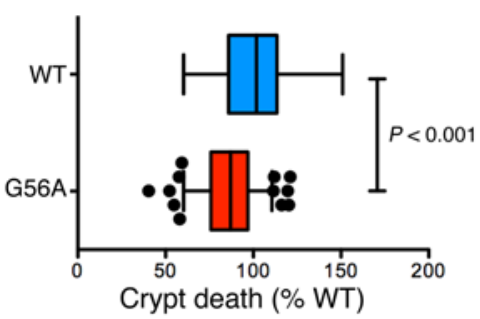

D

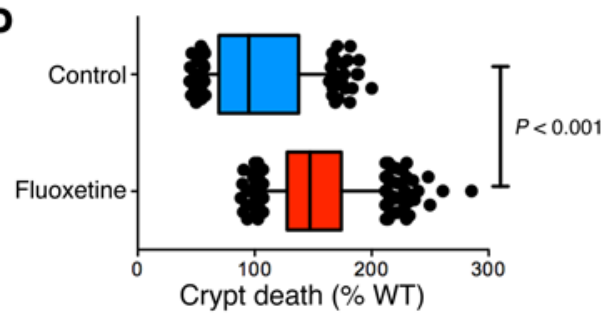

$\mathbf{F}$

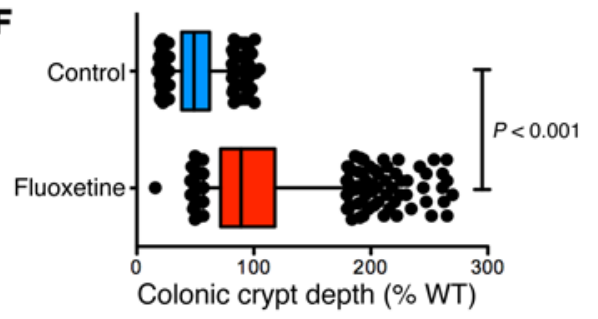

H

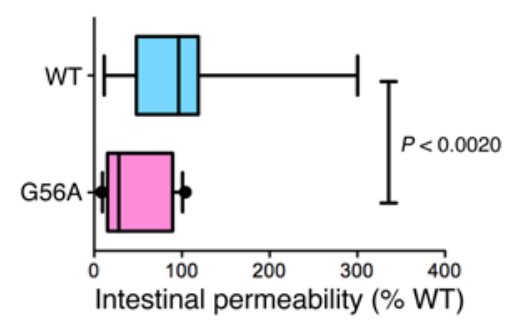

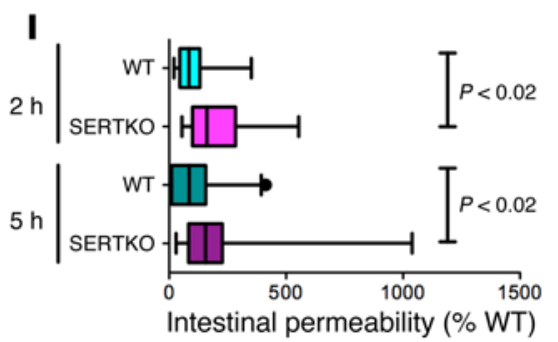

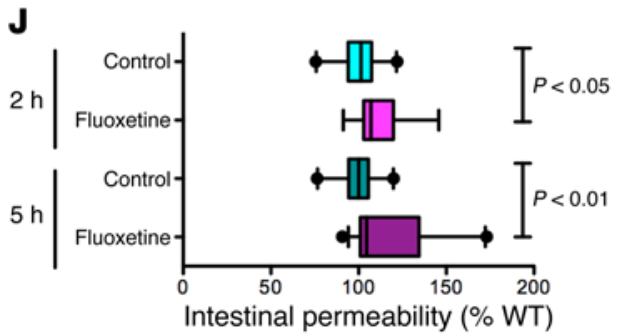

Figure 8. Effects of the SERT Ala56 mutation on mucosal growth and permeability. Effects of the SERT Ala56 mutation on mucosal growth $(n=6)$ and permeability (12-20/group, 2 trials) were reciprocal to those seen in SERTKO and fluoxetine-treated mice ( $n=4$ /group). (A, B, and E) Comparisons of SERT Ala56 mice with WT mice. (A) Villus height (small intestine). (B) Crypt depth (small intestine). (E) Crypt depth (colon). (G) Comparison of SERTKO with WT mice. (G) Crypt depth (colon). (C, D, and $\mathbf{F}$ ) Comparisons of fluoxetine-treated mice with control mice. (C) Villus height (small intestine). (D) Crypt depth (small intestine). (F) Crypt depth (colon). (H-J) Macromolecular permeability compared in SERT Ala56 (H), SERTKO (I), and fluoxetine-treated mice (J). Although macromolecular permeability in SERT Ala56 mice was significantly lower than that in WT animals $(\mathbf{H})$, permeability was increased at both 2 and 5 hours after gavage of fluorescent dextran in SERTKO and fluoxetine-treated mice. Student's unpaired $t$ test was used to compare groups. For the box-and-whisker plot, the boxes represent the first and third quartiles, the whiskers are $95 \%$ confidence interval, and the lines within the boxes are median values. and crypt depth (Figure 8D) were all significantly greater than those of WT littermates (Supplemental Figure 3, E and F). As previously reported (33), in SERTKO mice, small intestinal villus height $(142 \% \pm 6 \% \mathrm{WT} ; n=90$ sections from 6 mice) and crypt depth $(128 \% \pm 3 \% \mathrm{WT} ; n=90$ sections from 6 mice $)$ were significantly larger than in WT littermates. Similar results were obtained in measurements of colonic crypt depth, which was significantly shorter than WT in G56A mice (Figure 8E) but significantly larger than that of controls in fluoxetine-treated mice (Figure $8 \mathrm{~F}$ ) and larger than WT in SERTKO animals (Figure 8G).

Immunostaining of proliferating cells in crypts with Abs to Ki67 was used to verify whether altered proliferation of transit-amplifying cells was responsible for the differences observed in villus height and crypt depth. The mean number of Ki67- immunoreactive cells per small intestinal crypt $(26.1 \pm 0.7, n=30$ crypts) of SERT Ala56 mice was significantly less than that of WT mice (45.0 $\pm 4.2, n=31$ crypts; $P<0.001$; Supplemental Figure $3, \mathrm{C}$ and D). Similarly, in the colon, the mean number of Ki67immunoreactive cells per crypt ( $21.4 \pm 0.5, n=112$ crypts) of SERT Ala56 mice was significantly less than that of WT mice $(30.5 \pm 0.5$, $n=28$ crypts; $P<0.001)$. Crypt epithelial cells have previously been shown to proliferate more rapidly in SERTKO than in WT mice (33). Interestingly, proliferation in the intestinal crypts of fluoxetine-treated mice was similar to that previously observed in SERTKO animals and opposite to that of SERT Ala56 mice (Supplemental Figure 3, G-J). The mean number of Ki67-immunoreactive cells per small intestinal crypt $(22.3 \pm 0.3, n=78$ crypts $)$ of fluoxetine-treated mice was significantly greater than that of 
control animals $(14.7 \pm 00.3, n=31$ crypts; $P<0.001)$. Similarly, in the colon, the mean number of Ki67-immunoreactive cells per crypt (12.5 $\pm 0.3, n=80$ crypts) of fluoxetine-treated mice was significantly greater than that of control mice $(9.1 \pm 0.2, n=74$ crypts; $P<0.001)$. These observations support the hypothesis that mucosal growth is stunted in SERT Ala56 mice because SERT regulation of 5-HT signaling is important for epithelial proliferation and mucosal maintenance. The effects of SERT inhibition during development, moreover, are remarkably persistent, since the fluoxetine-treated animals were given at least 6 weeks after fluoxetine withdrawal for SERT activity to recover.

Macromolecular permeability was investigated to determine how the alterations in mucosal maintenance observed in SERT Ala56, SERTKO, and fluoxetine-treated mice affected the integrity of mucosal barrier function. Permeability to FITC-dextran in SERT Ala56 mice was found to be significantly less than that of WT animals (Figure $8 \mathrm{H}$ ). In contrast, permeability of the mucosa of SERTKO (Figure 8I) and fluoxetine-treated mice (Figure 8J) was more than that of WT animals. Electron microscopic analysis after i.v. injection of HRP failed to reveal a loss of tight junctional integrity in any of the mice tested (Supplemental Figure 1).

Transcription of Tph1 and Tph2 is abnormal when SERT activity is altered in developing bowel. Experiments were carried out to determine whether the hyperactive SERT of SERT Ala56 mice or its absence in SERTKO animals altered transcription of Tph1 and/ or Tph2. Results with SERTKO mice were compared with those obtained with fluoxetine-treated animals to distinguish long-lasting developmental effects from the acute consequences of the absence of SERT. In the intestines of SERT Ala56 mice, transcripts encoding TPH1 and TPH2 were each significantly more abundant than in those of WT animals (Supplemental Figure 2A). Interestingly, the relative density of EC cells and that of the entire enteroendocrine (EE) cell class were also significantly greater in SERT Ala56 than in WT mice (Supplemental Figure 2B). Because the majority of enteric TPH1 is located in EC cells, the high level of transcripts encoding Tph1 in SERT Ala56 mice is consistent with the greater abundance of EC cells in SERT Ala56 animals. In contrast, in the small and large intestines of SERTKO mice, transcripts encoding TPH1 and TPH2 were each significantly less abundant than in those of WT mice (Supplemental Figure 2C). Consistently, the relative density of EC cells and that of the entire EE cell class were also significantly lower in SERTKO than in WT mice (Supplemental Figure 2D). The effects of the SERT Ala56 mutation and SERTKO on transcription of Tph1 and Tph2, as well as on the relative abundance of EC and total EE cells, were thus the reverse of one another. In mice given fluoxetine throughout development, transcripts encoding TPH1 were significantly more abundant than in WT animals; however, no significant difference could be detected between fluoxetine-treated and control mice in transcripts encoding TPH2 (Supplemental Figure 2E). The significant decreases relative to WT in the EC and total EE cell density of fluoxetine-treated mice (Supplemental Figure 2, E and F) were similar to those observed in SERTKO animals. It is possible that the abundance of transcripts encoding TPH2 was depressed in SERTKO mice but not in fluoxetine-treated animals because the effect requires an ongoing deficiency of 5-HT inactivation. SERT was presumably not inhibited 6 weeks after fluoxetine withdrawal when animals were analyzed. Despite changes in abundance of transcripts encoding TPH1 and the density of EC cells, no significant change was seen in the concentration of 5-HT in the gut of either SERT Ala56 or fluoxetine-treated mice (data not shown). Secretion or intracellular metabolism of 5-HT, which were not measured, may thus balance changes in biosynthesis and uptake.

\section{Discussion}

SERT Ala56 is the most common of the rare hyperactive SERT variants that are overtransmitted to children with ASD $(10,11)$. SERT Ala56 mice exhibit hyperserotonemia and behaviors that align broadly with the core diagnostic features of $\operatorname{ASD}(12,13,45)$. These traits support the use of SERT Ala56 mice as a model for analyzing potential contributions of 5-HT signaling abnormalities to ASD. Both hyperserotonemia $(4,5)$ and GI dysfunction (2) are commonly seen in ASD. Because platelets derive their 5-HT from the gut (3) and 5-HT is an important multifunctional GI signaling molecule (17), we postulated that SERT hyperactivity would not only cause hyperserotonemia but also perturb GI structure and function in SERT Ala56 mice. Abnormal 5-HT signaling, moreover, impacts both CNS (38, 46-48) and ENS (22) development. We thus tested the hypothesis that ENS formation and ENS-dependent functions are perturbed in SERT Ala56 mice because of the reduction of endogenous 5-HT availability (13). To do so, we comprehensively analyzed SERT Ala56 mice and, for comparison, SERTKO and fluoxetine-treated animals, to provide, respectively, mice in which SERT is nonfunctional or is inhibited only during ontogeny. These studies of altered SERT activity also provided unique insights into roles of 5-HT signaling in enteric physiology and pathophysiology.

The ENS was strikingly hypoplastic in SERT Ala56 mice. Numbers of neurons were reduced in both plexuses of small and large intestines, and neurons generated after serotonergic neurons during ontogeny (expressing TH, CGRP, or GABA) were more deficient than ENS neurons in general. In contrast, the ENS of SERTKO and fluoxetine-treated animals was hyperplastic, and the late-born neurons that were deficient in SERT Ala56 mice were especially abundant in these animals. The similarity between SERTKO and fluoxetine-treated mice suggests that the developmental period is a critical time for SERT function in neurogenesis. These observations are consistent with the ideas that defective 5-HT signaling due to the increased 5-HT clearance of SERT Ala56 mice interferes with enteric neurogenesis, whereas potentiated serotonergic signaling due to the absence of SERT or its inhibition during development enhances neurogenesis. The sensitivity of late-born neurons to SERT activity is consistent with the ideas that serotonergic neurons, which are early-born, regulate enteric neurogenesis and thus help sculpt the ENS. These observations confirm that 5-HT is an ENS growth factor $(22,49)$ and that serotonergic signaling is essential for normal neurogenesis $(22,32)$. The data are also consistent with the hypothesis that a defect common to the ENS and CNS could be responsible in ASD for comorbid GI disturbances.

Motility of the SERT Ala56 bowel was impaired both in vivo (slowed GI transit time and colonic transit) and in vitro (decreased velocity, frequency, and length of conduction of CMMCs). Because CMMCs, which are ENS-dependent, were defective in isolated preparations of SERT Ala56 bowel, the motor abnor- 
mality is an intrinsic property of the ENS. This consideration is important because a global defect in SERT affects the CNS and well as the ENS. In both SERTKO and fluoxetine-treated mice, for example, the absence or inhibition of SERT during development was found to enhance sympathetic activity, which slowed GI motility in vivo. Both the ENS hypoplasia of SERT Ala56 mice and the hyperplasia seen in SERTKO and fluoxetine-treated animals thus have functional consequences.

Two subtypes of 5-HT receptor have been linked to 5-HT-promoted enteric neurogenesis, $5-\mathrm{HT}_{2 \mathrm{~B}}(49)$ and $5-\mathrm{HT}_{4}(32)$. Agonists of each promote enteric neurogenesis; moreover, postnatal enteric neurogenesis is defective when $5-\mathrm{HT}_{4}$ receptors are deleted (32). To test the hypothesis that $5-\mathrm{HT}_{4}$ receptors are critical in mediating the enteric neurodevelopmental actions of 5-HT, we determined whether a $5-\mathrm{HT}_{4}$ agonist, prucalopride (34), prevents the development of the SERT Ala56 ENS phenotype. Because prucalopride is not a substrate for SERT, its signaling should not be terminated prematurely, like that of 5-HT, by an overly active SERT. Prucalopride did, in fact, overcome the SERT Ala56 abnormality, preventing the ENS hypoplasia, deficiencies of late-developing neurons, the slowing of GI transit in vivo, and the defects in CMMCs that occur in vitro. Systemic agonist administration to dams precludes an absolute conclusion that the actions of prucalopride derive from direct stimulation of enteric neurogenesis in offspring, though this seems likely and is consistent with the known receptor contributions to developing enteric neurons. Regardless, the striking ability of prucalopride to prevent SERT Ala56-associated ENS neuronal hypoplasia and its consequences supports the idea that SERT and 5- $\mathrm{HT}_{4}$ receptors play important roles in 5-HTpromoted neurogenesis and also supports the overall hypothesis that the defects seen in SERT Ala56 mice are, in fact, due to the premature clearance of endogenous 5 -HT by the hyperactive SERT variant these animals express.

SERT has previously been shown to play a critical role in regulating the proliferation of crypt epithelial cells and mucosal maintenance (33). Serotonergic neurons, however, are restricted to the myenteric plexus and do not directly innervate the mucosa $(50,51)$; instead, myenteric serotonergic neurons innervate submucosal cholinergic neurons that provide a muscarinic input to the mucosa, which in turn stimulates epithelial proliferation and the growth of villi and crypts (33). Genetic deletion of SERT thus enhances the action of 5-HT at submucosal synapses, which indirectly increases villus height, crypt depth, and crypt epithelial proliferation. Conversely, the enhanced 5-HT clearance of SERT Ala56 mice leads to a reduction in villus height and crypt depth and causes the proliferation of crypt epithelium to be abnormally slow. The enhanced mucosal growth observed in fluoxetine-treated mice, which resembled that seen in SERTKO animals, reveals that a critical period exists for SERT's contribution to mucosal growth. SERT inhibition has long-term consequences even when, as in fluoxetine-treated mice, it is restricted to development. We propose that ENS hypoplasia due to enhanced SERT activity during development impairs mucosal maintenance during later life and suggest that mucosal maintenance is sustained by ongoing neuronal 5-HT signaling. It is also interesting that mucosal permeability to macromolecules appeared to be a direct function of mucosal growth. For example, macromolecular permeability was lowest in the hypoplastic mucosae of SERT Ala56 mice and greatest in the hyperplastic mucosae of SERTKO and fluoxetine-treated mice.

The SERT Ala56 mutation increased the GI expression of each of the 5-HT biosynthetic enzymes TPH1 and TPH2. These observations are consistent with the idea that the decreased availability of 5-HT in the SERT Ala56 bowel triggers a feedback increase in 5-HT biosynthetic capacity in both EC cells (TPH1) and neurons (TPH2). We also found that EC cells were more abundant in SERT Ala56 mice than in WT animals, which may account for the increased abundance of transcripts encoding TPH1. Feedback through 5-HT availability, however, may not account for the increase seen in total EE cells in SERT Ala56 animals, because the EE population includes cells that do not produce 5-HT. The diminished mucosal growth that occurs in SERT Ala56 animals may thus lead to an increase in the proportion of cells that differentiate along a neurogenin 3-dependent pathway and acquire an EE phenotype (52). In SERTKO mice, transcripts encoding TPH1 and TPH2 were each less abundant than in WT animals and the densities of both EC and total EE cells were decreased, a phenotype opposite to that seen in SERT Ala56 animals. These observations are consistent both with a feedback decrease in 5-HT biosynthesis due to enhanced 5-HT bioavailability in the absence of SERT and with a diminished acquisition of the EE phenotype when mucosal growth speeds up. The decrease in transcripts encoding TPH1 was mimicked in fluoxetine-treated mice, as was the decreased density of EC and total EE cells. Fluoxetine-treated mice, however, did not exhibit the decrease in abundance of transcripts encoding TPH2 that was seen in SERTKO animals; therefore, reduced TPH2 expression probably reflects the ongoing enhanced 5-HT bioavailability associated with SERT deletion. The mucosal effects of fluoxetine treatment, on the other hand, outlasted the period of acute SERT inhibition and are likely to be due to the rearranged ENS in these animals.

The GI impact of developmental administration of a SERT inhibitor persisted into adult life, long after the inhibitor was withdrawn. Depression occurs in up to $23 \%$ of pregnant women, and SERT inhibition is a common feature of most antidepressants used to treat it (15). Selective serotonin reuptake inhibitor (SSRI) use during pregnancy has been linked to a 2-fold increase in congenital defects (53). Children exposed to SSRIs or tricyclic antidepressants in utero were found to be 10 -fold more likely to require laxatives for constipation, which is consistent with the idea that antenatal SERT inhibition disturbs ENS development and leads to persistent defects in GI motility (54). The ENS contributes to many GI disorders in adults, including irritable bowel syndrome (55) and inflammatory bowel disease $(23,56)$. Given the importance of 5-HT signaling to ENS development and the long-lasting effects of fluoxetine treatment on sympathetic output, further investigation is probably warranted of the administration to pregnant or lactating women of antidepressants that affect SERT or 5-HT function.

ASD is severe in its prevalence, impact, and costs to society. Symptoms, more than etiology, define the syndrome; nevertheless, peripheral abnormalities, such as GI dysfunction (2), also occur. The complexity of the genetic contributions to ASD (57) encourages efforts to identify biomarkers, such as hyperserotonemia $(4,5)$, for convergent pathways to account for the pathophysiology. Because platelets acquire their 5-HT from the GI tract (3), 
the ASD-associated increase in platelet 5-HT links GI dysfunction to ASD. The gene encoding SERT (SLC6A4) has been associated with both platelet 5-HT content and susceptibility to ASD (58-61). The abnormalities of GI function that we have characterized in SERT Ala56 mice are also similar to those that occur at high frequency in $\operatorname{ASD}(2,62)$. Our observations thus support the idea that a defect in 5-HT-sensitive neurogenic pathways could be a common feature, describing a novel ASD subtype defined by "reverse phenotyping" (63), in which behavioral and enteric abnormalities due to abnormal serotonergic signaling underlie the condition. A prospective human study will be needed to confirm this suggestion. Interestingly, the $5-\mathrm{HT}_{4}$ receptor, which prucalopride rescue suggests is insufficiently stimulated in SERT Ala56 mice, also promotes hippocampal neurogenesis and has been postulated to underlie the neurogenesis induced by SSRIs (64). Prucalopride and other $5-\mathrm{HT}_{4}$ agonists were developed for treating chronic constipation and constipation-predominant irritable bowel syndrome (34). Whether $5-\mathrm{HT}_{4}$ agonists might also be helpful therapeutic agents for ASD requires further study.

\section{Methods}

Animals. C57BL/6 mice were used for studies of Slc6a4 deletion (SERTKO) and inhibition during development with fluoxetine. Fluoxetine $(7.5 \mathrm{mg} / \mathrm{kg} / \mathrm{d}$ ) was administered to pregnant dams from E1 (the time a mucous plug was detected) through weaning at P21. Animals were tested at 6-8 weeks of age. SERT Ala56 mice were generated on a $129 S 6$ background (12). Experiments were carried out with confirmed homozygous WT and SERT Ala56 littermates. The selective 5- $\mathrm{HT}_{4}$ agonist prucalopride (15 mg/kg; Shire Pharmaceuticals) was given to dams daily by gavage. Prucalopride was begun at E1 and was continued until P21. Control dams received autoclaved water, also by gavage. The E1-P21 period covers most of enteric neurogenesis (37). The effects of prucalopride were determined 3-5 weeks after cessation of therapy, ensuring that prucalopride itself was no longer present in the animals.

Immunocytochemistry. The immunoreactivities of the neuronal marker ANNA-1 (65-68) as well as tyrosine hydroxylase (TH), $\gamma$-aminobutyric acid (GABA), 5-HT, and calcitonin gene-related peptide (CGRP) were located to quantify the abundance of total, dopaminergic, GABAergic, 5-HT-expressing, and CGRP-expressing enteric neurons. Myenteric and submucosal plexuses were examined in whole mounts of laminar preparations of the gut wall. Methods used have been described previously $(22,23)$ and are detailed in Supplemental Methods.

Images were obtained with a cooled CCD camera and analyzed with computer assistance (Volocity 6.0 software, Improvision/PerkinElmer Life and Analytical Sciences). To count the numbers of labeled cells, a computer-controlled motorized stage was used to scan and collect images with a $\times 20$ objective covering the entirety of a $10-\mathrm{mm}^{2}$ area. Collected images were computer-processed (Volocity 6.0 software) to estimate numbers of immunoreactive cells of each type (cells per square millimeter of ganglionic area).
GI motility. Colonic motility was measured as the time required to expel a $3 \mathrm{~mm}$ glass bead inserted $2 \mathrm{~cm}$ into the rectum (69). Total GI transit time was estimated as the time required for a nonabsorbable dye (carmine red) to appear in stool after its gavage into the stomach (70). Gastric emptying and small bowel transit were evaluated following the gavage of rhodamine B dextran in methylcellulose. The gut was removed 15 minutes after gavage, and the percentage of dye remaining in the stomach as well as the geometric center of the rhodamine B dextran in the intestine was determined (71). Video imaging was used to analyze CMMCs in isolated colon $(72,73)$. Internal pressure was raised to initiate CMMCs, and spatiotemporal maps of colon diameter as a function of time were constructed. CMMC frequency, velocity, and length of conduction were measured $(74,75)$. See Supplemental Methods for further details.

Quantitation of transcripts. Methods used to extract RNA, reversetranscribe it to DNA, and quantify transcript abundance with real-time PCR have been described previously $(22,23)$ and are detailed in Supplemental Methods. Transcript expression was normalized to that of glyceraldehyde-3-phosphate dehydrogenase (Gapdh).

In vivo permeability. The absorption of FITC-dextran $(4.4 \mathrm{kDa}$; $22 \mathrm{mg} / \mathrm{ml}$ in PBS; Sigma-Aldrich) was evaluated by measurement of its concentration in blood (fluorescence at $520 \mathrm{~nm}$ ) after gavage (76). Submandibular puncture was used to obtain blood (100 $\mu \mathrm{l}) 2$ and 5 hours after the administration of FITC-dextran.

Statistics. Student's unpaired $t$ test and 1-way ANOVA were used, respectively, to compare single and multiple means. A $P$ value of less than 0.05 was considered significant.

Study approval. Animal studies were approved by the IACUC of Columbia University Medical Center.

\section{Author contributions}

KGM designed and conducted the studies, analyzed data, and wrote the manuscript. ZL, KS, VS, and NI conducted experiments and acquired data. GMA conducted experiments and acquired and analyzed data. IS acquired data. JV-V created and provided the Ala56 mice, conducted experiments, and analyzed data. RDB created and provided the Ala56 mice, conducted experiments, analyzed data, and critically reviewed the manuscript. MDG designed the studies, analyzed data, and wrote the manuscript.

\section{Acknowledgments}

This work was supported by NIH grants NS15547, DK093786, and MH096972, the Einhorn Charitable Trust, and the Autism Research Institute.

Address correspondence to: Kara Gross Margolis, Morgan Stanley Children's Hospital, Columbia University College of Physicians and Surgeons, Department of Pediatrics, Division of Pediatric Gastroenterology, 620 West 168th Street, New York, New York 10032, USA. Phone: 212.305.5903; E-mail: kjg2133@cumc.columbia.edu.
1. Posar A, Resca F, Visconti P. Autism according to diagnostic and statistical manual of mental disorders 5(th) edition: the need for further improvements. J Pediatr Neurosci. 2015;10(2):146-148.

2. McElhanon BO, McCracken C, Karpen S, Sharp WG. Gastrointestinal symptoms in autism spectrum disorder: a meta-analysis. Pediatrics. 2014;133(5):872-883.

3. Matondo RB, et al. Deletion of the serotonin transporter in rats disturbs serotonin homeostasis without impairing liver regeneration. Am J Physiol Gastrointest Liver Physiol.
2009;296(4):G963-G968.

4. Mulder EJ, et al. Platelet serotonin levels in pervasive developmental disorders and mental retardation: diagnostic group differences, within-group distribution, and behavioral correlates. J Am Acad Child Adolesc Psychiatry. 
2004;43(4):491-499.

5. Ritvo ER, Yuwiler A, Geller E, Ornitz EM, Saeger $\mathrm{K}$, Plotkin S. Increased blood serotonin and platelets in early infantile autism. Arch Gen Psychiatry. 1970;23(6):566-572.

6. Morrissey JJ, Walker MN, Lovenberg W. The absence of tryptophan hydroxylase activity in blood platelets. Proc Soc Exp Biol Med. 1977;154(4):496-499.

7. Lesch KP, Wolozin BL, Murphy DL, Riederer P. Primary structure of the human platelet serotonin (5-HT) uptake site: Identity with the brain 5-HT transporter. J Neurochem. 1993;60(6):2319-2322.

8. Hughes FB, Brodie BB. The mechanism of serotonin and catecholamine uptake by platelets. JPharmacol Exp Ther. 1959;127:96-102.

9. Prasad HC, et al. Human serotonin transporter variants display altered sensitivity to protein kinase $\mathrm{G}$ and $\mathrm{p} 38$ mitogen-activated protein kinase. Proc Natl Acad Sci US A. 2005;102(32):11545-11550.

10. Sutcliffe JS, et al. Allelic heterogeneity at the serotonin transporter locus (SLC6A4) confers susceptibility to autism and rigid-compulsive behaviors. Am J Hum Genet. 2005;77(2):265-279.

11. Prasad HC, Steiner JA, Sutcliffe JS, Blakely RD. Enhanced activity of human serotonin transporter variants associated with autism. Philos Trans $R$ Soc Lond B Biol Sci. 2009;364(1514):163-173.

12. Veenstra-Vanderweele J, et al. Modeling rare gene variation to gain insight into the oldest biomarker in autism: construction of the serotonin transporter Gly56Ala knock-in mouse. J Neurodev Disord. 2009;1(2):158-171.

13. Veenstra-VanderWeele J, et al. Autism gene variant causes hyperserotonemia, serotonin receptor hypersensitivity, social impairment and repetitive behavior. Proc Natl Acad Sci U S A. 2012;109(14):5469-5474.

14. Gaspar P, Cases O, Maroteaux L. The developmental role of serotonin: news from mouse molecular genetics. Nat Rev Neurosci. 2003;4(12):1002-1012.

15. Kepser LJ, Homberg JR. The neurodevelopmental effects of serotonin: a behavioural perspective. Behav Brain Res. 2015;277:3-13.

16. Liu C, Maejima T, Wyler SC, Casadesus G, Herlitze S, Deneris ES. Pet- 1 is required across different stages of life to regulate serotonergic function. Nat Neurosci. 2010;13(10):1190-1198.

17. Gershon MD. 5-Hydroxytryptamine (serotonin) in the gastrointestinal tract. Curr Opin Endocrinol Diabetes Obes. 2013;20(1):14-21.

18. Chen JJ, et al. Maintenance of serotonin in the intestinal mucosa and ganglia of mice that lack the high affinity serotonin transporter (SERT): abnormal intestinal motility and the expression of cation transporters. J Neurosci. 2001;21(16):6348-6361.

19. Wade PR, Chen J, Jaffe B, Kassem IS, Blakely $\mathrm{RD}$, Gershon MD. Localization and function of a 5-HT transporter in crypt epithelia of the gastrointestinal tract. J Neurosci. 1996;16(7):2352-2364.

20. Blakely RD, et al. Cloning and expression of a functional serotonin transporter from rat brain. Nature. 1991;354(6348):66-70.

21. Chen JX, Pan H, Rothman TP, Wade PR, Gershon MD. Guinea pig 5-HT transporter: cloning, expression, distribution and function in intestinal sensory reception. Am J Physiol. 1998;275(3 pt 1):G433-G448.

22. Li Z, et al. Essential roles of enteric neuronal serotonin in gastrointestinal motility and the development/survival of enteric dopaminergic neurons. J Neurosci. 2011;31(24):8998-9009.

23. Margolis KG, et al. Pharmacological reduction of mucosal but not neuronal serotonin opposes inflammation in mouse intestine. Gut. 2014;63(6):928-937.

24. Heredia DJ, Gershon MD, Koh SD, Corrigan $\mathrm{RD}$, Okamoto T, Smith TK. Important role of mucosal serotonin in colonic propulsion and peristaltic reflexes: in vitro analyses in mice lacking tryptophan hydroxylase 1. J Physiol. 2013;591(23):5939-5957.

25. Hillsley K, Grundy D. Sensitivity to 5-hydroxytryptamine in different afferent subpopulations within mesenteric nerves supplying the rat jejunum. J Physiol. 1998;509(pt 3):717-727.

26. Blackshaw LA, Grundy D. Effects of 5-hydroxytryptamine on discharge of vagal mucosal afferent fibres from the upper gastrointestinal tract of the ferret. J Auton Nerv Syst. 1993;45(1):41-50.

27. Lang IM. Noxious stimulation of emesis. Dig Dis Sci. 1999;44(8 suppl):58S-63S.

28. Pan H, Gershon MD. Activation of intrinsic afferent pathways in submucosal ganglia of the guinea pig small intestine. JNeurosci. 2000;20(9):3295-3309.

29. Bertrand PP, Kunze WAA, Furness JB, Bornstein JC. The terminals of myenteric intrinsic primary afferent neurons of the guinea pig ileum are excited by 5 -HT acting at 5-HT3 receptors. Neuroscience. 2000;101(2):459-469.

30. Bülbring E, Crema A. The release of 5-hydroxytryptamine in relation to pressure exerted on the intestinal mucosa. J Physiol. 1959;146(1):18-28.

31. Cooke HJ, Christofi FL. Enteric regulation of mucosal secretion. In: Johnson LR, ed. Physiology of the Gastrointestinal Tract. 4th ed. New York, New York, USA: Academic Press; 2006:737-762.

32. Liu MT, Kuan YH, Wang J, Hen R, Gershon MD. 5-HT4 receptor-mediated neuroprotection and neurogenesis in the enteric nervous system of adult mice. J Neurosci. 2009;29(31):9683-9699.

33. Gross ER, Gershon MD, Margolis KG, Gertsberg $\mathrm{ZV}$, Cowles RA. Neuronal serotonin regulates growth of the intestinal mucosa in mice. Gastroenterology. 2012;143(2):408-417.

34. Briejer MR, et al. The in vitro pharmacological profile of prucalopride, a novel enterokinetic compound. Eur J Pharmacol. 2001;423(1):71-83.

35. Karaosmanoglu T, Aygun B, Wade PR, Gershon MD. Regional differences in the number of neurons in the myenteric plexus of the guinea pig small intestine and colon: an evaluation of markers used to count neurons. Anat Rec. 1996;244(4):470-480.

36. Chalazonitis A, et al. Bone morphogenetic protein regulation of enteric neuronal phenotypic diversity: relationship to timing of cell cycle exit. J Comp Neurol. 2008;509(5):474-492.

37. Pham TD, Gershon MD, Rothman TP. Time of origin of neurons in the murine enteric nervous system. J Comp Neurol. 1991;314(4):789-798.

38. Bonnin A, et al. A transient placental source of serotonin for the fetal forebrain. Nature. 2011;472(7343):347-350.
39. Yamataka A, et al. Intestinal neuronal dysplasialike pathology in Ncx/Hox11L. JPediatr Surg. 2001;36(8):1293-1296.

40. Spencer NJ, Bywater RA, Taylor GS. Evidence that myoelectric complexes in the isolated mouse colon may not be of myogenic origin. Neurosci Lett. 1998;250(3):153-156.

41. Misiewicz JJ, Waller SL, Eisner M. Motor responses of human gastrointestinal tract to 5 -hydroxytryptamine in vivo and in vitro. Gut. 1966;7(3):208-216.

42. Oosterbosch L, von der Ohe M, Valdovinos MA, Kost LJ, Phillips SF, Camilleri M. Effects of serotonin on rat ileocolonic transit and fluid transfer in vivo: possible mechanisms of action. Gut. 1993;34(6):794-798.

43. Adamec R, Burton P, Blundell J, Murphy DL, Holmes A. Vulnerability to mild predator stress in serotonin transporter knockout mice. Behav Brain Res. 2006;170(1):126-140.

44. Smith TK, Gershon MD. CrossTalk proposal: 5-HT is necessary for peristalsis. J Physiol. 2015;593(15):3225-3227.

45. Kerr TM, et al. Genetic background modulates phenotypes of serotonin transporter Ala56 knock-in mice. Mol Autism. 2013;4(1):35.

46. Kawasaki H. Spatio-temporal regulation of the formation of the somatosensory system. Dev Growth Differ. 2015;57(3):193-199.

47. Chen X, Ye R, Gargus JJ, Blakely RD, Dobrenis $\mathrm{K}$, Sze JY. Disruption of transient serotonin accumulation by non-serotonin-producing neurons impairs cortical map development [published online ahead of print January 14, 2015]. Cell Rep. doi:10.1016/j.celrep.2014.12.033.

48. Deneris ES. Molecular genetics of mouse serotonin neurons across the lifespan. Neuroscience. 2011;197:17-27.

49. Fiorica-Howells E, Maroteaux L, Gershon MD. Serotonin and the 5-HT(2B) receptor in the development of enteric neurons. J Neurosci. 2000;20(1):294-305.

50. Furness JB, Costa M. Neurons with 5-hydroxytryptamine-like immunoreactivity in the enteric nervous system: Their projections in the guinea pig small intestine. Neuroscience. 1982;7(2):341-350.

51. Qu ZD, Thacker M, Castelucci P, Bagyanszki M, Epstein ML, Furness JB. Immunohistochemical analysis of neuron types in the mouse small intestine. Cell Tissue Res. 2008;334(2):147-161.

52. Clevers H. The intestinal crypt, a prototype stem cell compartment. Cell. 2013;154(2):274-284.

53. Wogelius P, et al. Maternal use of selective serotonin reuptake inhibitors and risk of congenital malformations. Epidemiology. 2006;17(6):701-704.

54. Nijenhuis CM, ter Horst PG, van Rein N, Wilffert B, de Jong-van den Berg LT. Disturbed development of the enteric nervous system after in utero exposure of selective serotonin re-uptake inhibitors and tricyclic antidepressants. Br J Clin Pharmacol. 2012;73(1):126-134.

55. Gershon MD. Nerves, reflexes, and the enteric nervous system: pathogenesis of the irritable bowel syndrome. J Clin Gastroenterol. 2005;39(5 suppl 3):S184-S193.

56. Margolis KG, et al. Enteric neuronal density contributes to the severity of intestinal inflammation. Gastroenterology. 2011;141(2):588-598. 
57. Persico AM, Napolioni V. Autism genetics. Behav Brain Res. 2013;251:95-112.

58. Devlin B, et al. Autism and the serotonin transporter: the long and short of it. Mol Psychiatry. 2005;10(12):1110-1116.

59. Huang CH, Santangelo SL. Autism and serotonin transporter gene polymorphisms: a systematic review and meta-analysis. Am JMed Genet B Neuropsychiatr Genet. 2008;147B(6):903-913.

60. Kistner-Griffin E, Brune CW, Davis LK, Sutcliffe JS, Cox NJ, Cook EH Jr. Parent-of-origin effects of the serotonin transporter gene associated with autism. Am JMed Genet B Neuropsychiatr Genet. 2011;156(2):139-144.

61. Veenstra-VanderWeele J, et al. Transmission disequilibrium studies of the serotonin 5-HT2A receptor gene (HTR2A) in autism. Am JMed Genet. 2002;114(3):277-283.

62. Hsiao EY. Gastrointestinal issues in autism spectrum disorder. Harv Rev Psychiatry. 2014;22(2):104-111.

63. Schulze TG, McMahon FJ. Defining the phenotype in human genetic studies: forward genetics and reverse phenotyping. Hum Hered. 2004;58(3-4):131-138.

64. Imoto Y, et al. Role of the 5-HT4 receptor in chronic fluoxetine treatment-induced neurogenic activity and granule cell dematuration in the dentate gyrus. Mol Brain. 2015;8:29.

65. King PH, Redden D, Palmgren JS, Nabors LB, Lennon VA. Hu antigen specificities of ANNA-I autoantibodies in paraneoplastic neurological disease. J Autoimmun. 1999;13(4):435-443.

66. Lennon VA. The case for a descriptive generic nomenclature: clarification of immunostaining criteria for PCA-1, ANNA-1, and ANNA-2 autoantibodies. Neurology. 1994;44(12):2412-2415.

67. Chu G, Wilson PC, Carter CD, Lennon VA, Roberts-Thomson IC. Intestinal pseudo-obstruction, type 1 anti-neuronal nuclear antibodies and small-cell carcinoma of the lung. J Gastroenterol Hepatol. 1993;8(6):604-606.

68. Chalk CH, Lennon VA, Stevens JC, Windebank AJ. Seronegativity for type 1 antineuronal nuclear antibodies ('anti-Hu') in subacute sensory neuronopathy patients without cancer. Neurology. 1993;43(11):2209-2211.

69. Li ZS, Schmauss C, Cuenca A, Ratcliffe E, Gershon MD. Physiological modulation of intestinal motility by enteric dopaminergic neurons and the D2 receptor: analysis of dopamine receptor expression, location, development, and function in wild-type and knock-out mice. J Neurosci. 2006;26(10):2798-2807.

70. Kimball ES, Palmer JM, D’Andrea MR, Hornby PJ, Wade PR. Acute colitis induction by oil of mustard results in later development of an IBS-like accelerated upper GI transit in mice. Am JPhysiol Gastrointest Liver Physiol. 2005;288(6):G1266-G1273.

71. Miller MS, Galligan JJ, Burks TF. Accurate measurement of intestinal transit in the rat. JPharmacol Methods. 1981;6(3):211-217.

72. Roberts RR, Bornstein JC, Bergner AJ, Young HM. Disturbances of colonic motility in mouse models of Hirschsprung's disease. Am J Physiol Gastrointest Liver Physiol. 2008;294(4):G996-G1008.

73. Roberts RR, Murphy JF, Young HM, Bornstein JC. Development of colonic motility in the neonatal mouse-studies using spatiotemporal maps. Am J Physiol Gastrointest Liver Physiol. 2007;292(3):G930-G938.

74. Gwynne RM, Thomas EA, Goh SM, Sjovall H, Bornstein JC. Segmentation induced by intraluminal fatty acid in isolated guinea-pig duodenum and jejunum. J Physiol. 2004;556(pt 2):557-569.

75. Welch MG, Margolis KG, Li Z, Gershon MD. Oxytocin regulates gastrointestinal motility, inflammation, macromolecular permeability, and mucosal maintenance in mice. Am J Physiol Gastrointest Liver Physiol. 2014;307(8):G848-G862.

76. Chaniotou Z, et al. Corticotropin-releasing factor regulates TLR4 expression in the colon and protects mice from colitis. Gastroenterology. 2010;139(6):2083-2092. 